FRANZ-XAVER KAUFMANN

\title{
Sozialpolitik zwischen Gemeinwohl und Solidarität
}

Das Forschungsprojekt „Gemeinwohl und Gemeinsinn“ der Berlin-Brandenburgischen Akademie der Wissenschaften nimmt klassische Begriffe der politischen Theorie auf, um sie für neue Fragestellungen fruchtbar zu machen, wie sie vor allem aus der Perspektive des amerikanischen Kommunitarismus aufgeworfen worden sind. Den Kontext bildet ein verbreitetes Unbehagen über mutmaßliche Auswirkungen von zumeist unter dem Begriff der Globalisierung zusammengefaßten Phänomenen, unter denen die weitestgehende Entgrenzung und technische Beschleunigung des Wirtschaftsverkehrs und das Zusammenwachsen bisher getrennter Finanzmärkte zu einem tendenziell weltweiten, ausschließlich geldvermittelten Regelungszusammenhang zunächst wohl die folgenreichsten sind. Auf der Ebene des öffentlichen Bewußtseins fällt der Einflußgewinn eines von den Vereinigten Staaten ausgehenden individualistischen Paradigmas der Wirtschafts- und Sozialwissenschaften sowie die Infragestellung des bisherigen nationalstaatlich verfaßten Gesellschaftsbewußtseins durch die Vorstellung einheitlicher Weltzusammenhänge ins Gewicht. Diese kritische Bewußtseinslage ähnelt in vielem derjenigen um die Mitte des 19. Jahrhunderts, als die Wucht von Industrialisierung und Verstädterung die bisher dominierenden ländlichen Lebens- und Wirtschaftsformen verdrängte und die herkömmlichen Sorgeverbände in Frage stellte. Die Defizite der neuen Vergesellschaftungsform wurden damals unter der Semantik des Sozialen thematisiert, und so entstand - zuerst im deutschen Sprachraum - der Begriff der Sozialpolitik zur Kennzeichnung der Vorschläge, die dem Staate als Reaktion auf die Desorganisierung der hergebrachten Lebenszusammenhänge von sozialwissenschaftlicher Seite nahegelegt wurden. Ein vergleichbarer Diskurs entstand in Frankreich unter dem Signum der , solidarité

Der folgende Beitrag geht von dieser Analogie der Situationen aus. Auch heute erscheinen gewachsene politische und soziale Zusammenhänge durch neue technische und ökonomische Entwicklungen bedroht. Und wiederum werden sozialmoralische Postulate wie ,Gemeinwohl' und ,Gemeinsinn' bemüht, um Gegengewichte zu schaffen. Der Beitrag hat demzufolge zwei Teile: Er beginnt mit einem historischen Rückblick auf die sozialwissenschaftliche Verarbeitung der Umbruchsituation um die Mitte des 19. Jahrhunderts und verweist auf einige tragende Ideen der daraus entstehenden Sozi- 
alpolitik, sowie deren Wandel. Dabei soll deutlich werden, daß die nationalistisch inspirierten gesellschaftsintegrativen Intentionen von Sozialpolitik bereits auf einer reduktionistischen Problembestimmung beruhen, die den zuerst von Hegel auf den Begriff gebrachten strukturellen Differenzierungen des umfassenden Sozialzusammenhangs als ,Staat", ,bürgerliche Gesellschaft" und ,Familie' nicht gerecht werden. Luhmann hat diese Einsicht zu Ende gedacht, aber gleichzeitig die Funktion sozialmoralischer Ressourcen ungebührlich minimiert. Seine Theorie ist eine Gesellschaftstheorie, jedoch keine Theorie des Sozialen. Im zweiten Teil wird deshalb die gegenwärtige Renaissance des sozialmoralischen Denkens ernst genommen, jedoch gleichzeitig der dabei meist implizierte direkte Zusammenhang zwischen der Regeneration sozialmoralischer Ressourcen (,Gemeinsinn') und der Orientierung gesellschaftlicher Zusammenhänge (,Gemeinwohl ${ }^{\circ}$ ) problematisiert. Das herkömmliche politische Denken ist der im Zuge der Globalisierung zu beobachtenden Vervielfältigung der Solidaritätshorizonte nicht gewachsen. Als analytisches Konzept zum besseren Verständnis dieser Problematik wird anstelle des unterkomplexen Gemeinschaftskonzeptes ein Konzept von Solidarität als spezifischem Modus der Handlungskoordination vorgeschlagen, das sich unterschiedlich für traditional bestimmte und für typisch moderne Sozialzusammenhänge auslegen läßt.

\section{Soziale Frage und Sozialpolitik als Interpretationsrahmen der Krisenbewältigung im Zuge der Industrialisierung}

Denkmuster der Politikwissenschaft lassen sich bis zur Polis-Philosophie der alten Griechen zurückverfolgen. Die politische Philosophie der frühen Neuzeit basierte auf dem aristotelischen Verständnis einer politisch verfaßten Gesellschaft, welches sich dem aufkommenden nationalstaatlichen Denken als durchaus kongenial erwies. Dieses Politikmodell bildet auch den Kontext für den zeitgenössischen Diskurs eines strukturellen Zusammenhangs von Gemeinwohl und Gemeinsinn. 'Die Sozialwissenschaften dagegen sind erst im Rahmen der Auseinandersetzung mit dem großen Umbruch entstanden, der in knappster Form durch die kulturellen Transformationen der Aufklärung, die ökonomisch-technischen Transformationen von Freihandel und Industrialisierung sowie die politischen Transformationen von Konstitutionalismus und Demokratisierung zu kennzeichnen ist. Die Sprengung des durch die statischen Momente des Bodens und der Tradition geprägten ,Ancien Régime ${ }^{6}$ und der Aufbruch in ein ,historisches ${ }^{6}$, d.h. durch Krise und Wandel gekennzeichnetes Zeitalter rief nach neuen, komplexeren Interpretationsmustern menschlichen Zusammenlebens, die sich im französischen und deutschen Sprachraum mit dem Aufkommen des Wortes, Sozial' verbanden. ${ }^{2}$

Vgl. Münkler/Fischer 1999, S. 241 ff.

Für Frankreich vgl. Donzelot 1984; für Deutschland Pankoke 1970. Die Ausführungen in Abschnitt 1 beruhen auf einer umfangreicheren begriffsgeschichtlichen Studie, vgl. Kaufmann 2001. 


\subsection{Soziale Politik als Vermittlung zwischen ,Staat ${ }^{6}$ und ,Bürgerlicher Gesellschaft ${ }^{\star}$}

Die Semantik des Sozialen im Unterschied zum Politischen reflektiert den Prozeß einer Differenzierung und Verselbständigung unterschiedlicher Lebensbereiche, den als erster Hegel auf die begrifflichen Unterscheidungen von ,Staat", ,Bürgerlicher Gesellschaft ${ }^{6}$ und ,Familie' gebracht hat. Während im angelsächsischen Raum bis in jüngste Zeit ein aristotelisches Verständnis von Gesellschaft als politisch konstituierter Einheit vorherrschte, ${ }^{3}$ und auch in Frankreich Rousseaus Idee des Gesellschaftsvertrages den Unterschied zwischen dem Politischen und dem Sozialen zunächst noch latent hielt, hat Hegel die millenäre Vorstellung einer durch politische Herrschaft konstituierten gesellschaftlichen Einheit in die Differenz von Staat, bürgerlicher Gesellschaft und Familie aufgelöst. Dies stellte den Anfang einer Theorie funktionaler Differenzierung moderner Gesellschaften dar, welche insbesondere durch Talcott Parsons und Niklas Luhmann zu einem der bedeutendsten Paradigmen moderner Gesellschaftstheorie geworden ist.

Nicht nur in der englischen und französischen Aufklärung, auch in Deutschland hatte sich im Gefolge von Kant und Fichte eine stark individualistische Auffassung von Politik und Wirtschaft durchgesetzt. Dem gegenüber finden wir bei Hegel die gesellschaftstheoretischen Grundlage für das, was später unter dem Namen ,Sozialpolitik' seinen Begriff fand. Die menschliche Sittlichkeit äußert sich im Staate nach Hegel als freie Bejahung einer die Freiheit auch der Mitmenschen sichernden Rechtsordnung und findet in der Familie die sittliche Bedingung ihrer Entfaltung. In der bürgerlichen Gesellschaft dagegen äußert sie sich als ungebundener Wille zur Befriedigung von Bedürfnissen. Dieser Wille verwirklicht sich als Arbeit, die unter den Bedingungen der bürgerlichen Gesellschaft vornehmlich zur Arbeit für die Befriedigung der Bedürfnisse anderer als Bedingung der Befriedigung eigener Bedürfnisse wird. Im Unterschied zur ökonomischen Theorie der Briten konzeptualisierte Hegel somit die ökonomischen Verhältnisse nicht als durch Marktpreise vermittelte Tauschprozesse, sondern als moralisches Verhältnis zwischen Individuen. Allerdings insistierte Hegel auf dem ,negativen“ Charakter dieser Moralität. Die Willkür als Handlungsprinzip in der bürgerlichen Gesellschaft ist zwar Ausdruck menschlicher Freiheit, sie steht jedoch gleichzeitig in Spannung zu den sittlichen Prinzipien des Staates und der Familie. Die Bedürfnisse des Wirtschaftsbürgers führen nicht für sich allein zu einem, „System der Bedürfnisse“, wie es die bürgerliche Gesellschaft darstellt. Vielmehr wird die wechselseitige Vorteilhaftigkeit der „Vermittelung des Bedürfnisses" erst durch die Gewährleistung des Privateigentums und die Rechtssicherheit der Vertragsverhältnisse, also durch Leistungen des Staates hergestellt, dessen Konstitution als Verfassungsstaat Hegel als die geschichtliche Verwirklichung der Vernunft begriff. ${ }^{4}$ Das Individuum wird somit in der Hegel-

\footnotetext{
3 Erst der in Auseinandersetzung mit der postsozialistischen Situation in Osteuropa entstandene Diskurs über ,Civil Society schärft auch im angelsächsischen Raum den Sinn für die Differenz zwischen dem Politischen und dem Sozialen. Einen Überblick über die internationale Diskussion gibt Berger 1997.

4 Vgl. Siep 1992.
} 
schen Gesellschaftstheorie im doppelten Sinne zum Bürger: als Citoyen im Staate und als Bourgeois in der bürgerlichen Gesellschaft.

Hegel betonte die „Zufälligkeit und Willkür" der Verhältnisse in der bürgerlichen Gesellschaft. Diese sei kein Naturzustand, sondern das Ergebnis der eigennützigen Interessenverfolgung willkürlich handelnder Individuen. Im Unterschied zu den grundsätzlich gleichen Bürgerrechten im Verfassungsstaat resultiert in der bürgerlichen Gesellschaft aus der „Ungleichheit der Geschicklichkeit, des Vermögens und selbst der intellektuellen und moralischen Bildung" der Individuen eine „in der Idee enthaltene [...] Ungleichheit der Menschen“, und dieser ,die Forderung der Gleichheit entgegensetzen, gehört dem leeren Verstande an [...]." "Die soziale Ungleichheit der Personen ist somit für Hegel ein konstitutives Moment der bürgerlichen Gesellschaft. Es ist Aufgabe des Staates, für das erforderliche Maß an Gleichheit zu sorgen, das jedoch bei Hegel nicht näher bestimmt wird.

Die Hegelsche Gesellschaftsdiagnose wurde in der Folge von Karl Marx und Lorenz von Stein in klassentheoretischer Perspektive aufgenommen und mit den Diagnosen der Frühsozialisten auf unterschiedliche Weise verknüpft. Für die Geschichte der Sozialpolitik und die Entstehung des Sozialstaatsgedankens wurde Lorenz von Stein maßgeblich, der bereits als Konsequenz seiner Auseinandersetzung mit den sozialistischen und kommunistischen Strömungen in Frankreich die Forderung nach einem ,Königtum der sozialen Reform' erhoben hatte, das den Klassengegensatz im Sinne eines produktiven Kompromisses überwinden könne. Aufgabe des Staates sei es, einerseits die Eigentumsrechte für die besitzende Klasse zu gewährleisten und andererseits die Emanzipation der arbeitenden Klasse durch eine das Bildungswesen einschließende ,soziale Verwaltung direkt, und durch die Ermöglichung freier Assoziation im Sinne einer, sozialen Bewegung ‘ indirekt zu fördern..$^{6}$ Es ist also der Staat, der durch eine „Politik der sozialen Reform" die Antagonismen der bürgerlichen Gesellschaft in produktive Bahnen zu lenken vermag.

\subsection{Das ,Soziale‘ als Postulat der Überwindung einer Defiziterfahrung}

Die „Lehre von der Gesellschaft“ wurde zum zentralen Thema der entstehenden Sozialwissenschaften, wobei es ein Charakteristikum der deutschen Tradition gewesen ist, ,Gesellschaft' im Unterschied zum Staate als intermediären Bereich zu bestimmen, hierunter also vor allem Korporationen, freie Assoziationen, Stände, soziale Klassen und ähnliche Formationen ,zwischen ${ }^{6}$ Individuum und Staat zu verstehen. ${ }^{7}$ Diese Auffassung entwikkelte sich in Kritik an den französischen Verhältnissen, wo im Zuge der Revolution alle intermediären Instanzen zwischen Zentralstaat und Individuum, also z.B. auch Gewerkschaften, für illegal erklärt worden waren, was aus deutscher Sicht als schrankenloser Individualismus erschien. In mancherlei Hinsicht liest sich die heutige Kritik der sog.

\footnotetext{
Hegel 1955, \$200.

Stein 1850 , Bd. 3.

Eine vorzügliche, kommentierte Textsammlung bietet Pankoke 1991.
} 
Kommunitaristen ${ }^{8}$ am amerikanischen Individualismus wie eine Reprise der deutschen Diskussion um die Mitte des 19. Jahrhunderts.

Allein die deutsche Sprache kennt die semantische Unterscheidung von ,gesellschaftlich' und ,sozial' ${ }^{\circ}$. Die beiden Bezeichnungen haben sich zwar nicht in einer begrifflichen Differenz verfestigt, doch wurde, gesellschaftlich` zumeist deskriptiv mit den immer weiträumigeren und anonymeren Beziehungsnetzen assoziiert, während ,sozial' eher die moralischen Aspekte menschlichen Zusammenlebens abdeckte. ${ }^{9}$ Die Unterscheidung zwischen, politisch' und, sozial' entwickelte sich im Vormärz, vor allem aber unter dem Eindruck des Scheiterns der Zweiten Republik in Frankreich (1848). Die Verweigerung des von den Sozialisten geforderten ,Rechtes auf Arbeit" und die blutige Unterdrückung der auf die Schließung der ,Ateliers Nationaux‘ folgenden Arbeiteraufstände ließen erstmals den Konflikt innerhalb des demokratischen Staates, also unter den ,Citoyens' aufbrechen. Nun wurde deutlich, daß die Erlangung der politischen Rechte noch keine Verbesserung der sozialen Lage für die Arbeiter mit sich brachte. So formulierte schon 1845 noch unter den Bedingungen des Zensuswahlrechts der aus Deutschland exilierte Handwerker Karl Grün: „Was gibt die Konstitution dem Volke? Rechte, aber kein Brot, keine Arbeit, keine Erziehung. Rechte sind Steine, und diese Steine erhalten nicht einmal alle; wer im niedrigsten Falle keine 20 Gulden Steuern bezahlt, wer also nicht schon Brot hat, erhält nicht einmal die Steine des Rechts." ${ }^{10}$

Das Problem wurde also nicht im Staate, sondern in den Wirkungszusammenhängen der bürgerlichen Gesellschaft lokalisiert und als Soziale Frage bezeichnet. Dies war der aus dem Französischen (,question sociale‘) übernommene Name für die Folgeprobleme einer Auflösung der feudalen Ordnung: Pauperismus, Landflucht, Verstädterung, sowie das Elend der Frühindustrialisierung. Nur Karl Marx und Lorenz von Stein haben allerdings die Tragweite dieser, gesellschaftlichen' Veränderungen als dynamischen Prozeß eines potentiell destruktiven Klassenkampfes auf den Begriff gebracht. Recht verbreitet war dagegen die Vorstellung, daß es nur durch , aufgeklärte" staatliche Eingriffe zu einer Verbesserung der sozialen Verhältnisse bzw. einer ,Lösung der sozialen Frage ${ }^{\natural}$ kommen könne. Die hierzu erforderliche sozialwissenschaftliche Aufklärung wurde in Deutschland ab ca. 1848 als Aufgabe von Sozialpolitik bezeichnet:

„Der Inbegriff der Verhältnisse, nach welchen in einem Volke die Vorzüge der Geburt, des Besitzes und der Einsicht, so wie das auf diesen Vorzügen beruhende Ansehen verteilt ist, heißt die Gesellschaft. Bis zum Beginn des laufenden Jahrhunderts hatte man diese Verhältnisse nie zum Gegenstande der wissenschaftlichen Betrachtung gemacht. Seitdem die Wissenschaft sich dieses Gegenstandes einmal bemächtigt, kann sie ihn nie wieder aufgeben. Wir leben als Bürger zweier Welten, des Staates und der Gesellschaft. Das Verhältnis dieser beiden

8 Hierbei handelt es sich um eine in sich keineswegs homogene Bewegung; vgl. als Überblicke Zahlmann 1992; Brumlik/Brunkhorst 1993; Honneth 1993.

9 In dieser Funktion wurde das zu unspezifische, sozial' in der Folge durch andere Begriffe abgelöst, insbesondere durch, gemeinschaftlich" und, solidarisch" in ihrer substantivierten Form. Siehe dazu Abschnitt 2.

${ }^{10}$ Grün 1974, S. 19. 
Welten zu einander zu bestimmen, wird in alle Zukunft eine Hauptaufgabe der politischen Forschung sein. Es kann in Zukunft keine andere Politik geben als Sozialpolitik."

Sozialpolitik wurde hier als eine gesellschaftsgestaltende Politik begründende Sozialwissenschaft verstanden, welche ihren Ausgangspunkt von einem Studium der sozialen Verhältnisse unter dem Gesichtspunkt ihrer verfestigten Verteilungsmuster nimmt.

Worin aber bestand ,das Soziale' dieser Politik? Der Begriff hatte keinen eindeutigen Inhalt, setzte sich aber kritisch von einer ausschließlich nach den Prinzipien des Individualismus gestalteten ,Gesellschaft' ab, wobei bald die Erinnerung an geruhsamere und fürsorglichere Umstände der ständisch-feudalen Ordnung, bald die Antizipation einer besseren zukünftigen Gesellschaft den Horizont der Kritik bildeten. Das Soziale wurde zunächst ex negativo bestimmt; es ist das, was einer voll individualisierten Gesellschaft im Sinne des Programms der französischen Revolutionsverfassung fehlt. ${ }^{12}$ Deutlich unterschieden sich die politisch-ideologischen Positionen hinsichtlich der Lösungsvorschläge der ,Sozialen Frage': Die Konservativen versprachen sich eine Lösung von der Wiederherstellung ständischer Einordnungen und eines traditionellen Ethos; die Liberalen stellten eine Lösung durch den Fortschritt der Industrialisierung und eine verbreitete Eigentumsbildung in Aussicht; die radikalen Sozialisten versprachen sich eine Lösung von der Aufhebung des Privateigentums und der daraus folgenden Gleichheit; die Sozialreformer schließlich setzten auf die Selbstorganisation der Arbeiter und auf staatliche Maßnahmen zu deren Schutz und Förderung.

Betrachten wir diese erste Entwicklungsphase des Begriffs Sozialpolitik in der Perspektive von Gemeinwohl und Gemeinsinn, so fällt das Fehlen moralischer Appelle oder solidaritätsorientierter Argumentationen auf. Sozialpolitik wurde im wesentlichen als Problem der wissenschaftlichen Aufklärung eines problematischen gesellschaftlichen Zustandes und der Empfehlung politischer Mittel zu seiner Behebung betrachtet. Lorenz von Stein z.B. setzte nicht auf Gemeinsinn, sondern auf Einsicht in die wechselseitige Abhängigkeit von Kapital und Arbeit und auf staatliche Vermittlung. Im Sinne der Gemeinwohlrhetorik erscheint hier die Feststellung des Gemeinwohls als wissenschaftliche Aufgabe, und die Gemeinwohlorientierung wird als dem Staate implizit im Sinne des Hegelschen Begriffs vorausgesetzt. Zugleich wird für den Bereich der bürgerlichen Gesellschaft die Verfolgung des Eigeninteresses nicht in Frage gestellt, dem ,Bourgeois“ also kein ,Gemeinsinn“ abverlangt. Das „moderne Trennungsdenken " (Pankoke) löste mit der Leitvorstellung der Polis auch den Zusammenhang zwischen Gemeinsinn und Gemeinwohl auf.

Natürlich implizierte die Diagnose einer ,sozialen Frage" auch moralische Gesichtspunkte, aber diese wurden kaum explizit gemacht. Nahezu allgemein war die Einschätzung, daß die gesellschaftlichen Zustände des Pauperismus und der Frühindustrialisierung unerträglich seien, und dies verdient immerhin Beachtung, wenn wir an die Verhältnisse in großen Teilen der Dritten Welt und selbst in den Vereinigten Staaten denken. Die Verelendung der Unterschichten fand in Europa keine Legitimation, sondern fortgesetzte Kritik und vielfach engagierte Hilfe. Wir dürfen hier die Wirksamkeit

\footnotetext{
11 Meyer 1864, S. 319.

12 In diesem Sinne auch Donzelot 1984.
} 
eines Ethos annehmen, das sowohl von den Traditionen des Christentums als auch vom Humanismus der Aufklärung geformt worden war. Sowohl religiöse Bewegungen als auch individuelle , moralische Unternehmer' nahmen sich schon vor der Politik der Nöte der Unterschichten an. ${ }^{13}$ Eduard Heimann führte die Überwindbarkeit des Klassenkampfes auf dieses dem Bürgertum und der Arbeiterschaft gemeinsame Ethos zurück, dem die ,soziale Idee' entsprang, welche sich in der ,sozialen Bewegung' Ausdruck verschaffte. Indem die sozialistische Arbeiterbewegung als Speerspitze der sozialen Bewegung die kapitalistische Ordnung bedrohte, ermöglichte sie Sozialpolitik, welche, indem sie der sozialen Forderung nachgibt, den Kapitalismus stückweise abbaut und dadurch seinen jeweils verbleibenden Rest rettet. Die Pointe dieser Argumentation ist jedoch: Die soziale Idee motiviert zwar die Kritik an den herrschenden Zuständen und die Suche nach sozialen Reformen, diese vermögen sich jedoch nur insoweit durchzusetzen, als sie sich auch als wirtschaftlich zweckmäßig, ,produktionspolitisch notwendig" erweisen. ${ }^{14}$ Während somit der ältere Diskurs Sozialpolitik auf den Staat als handelnden Akteur bezog, sah Heimann unter den Bedingungen der Weimarer Zeit ${ }^{15}$ Sozialpolitik als unmittelbares Ergebnis der Arbeiterbewegung. Dem Staate wies Heimann nur eine instrumentelle Rolle zu, worin er dem Marxschen Denkschema folgte.

\subsection{Sozialpolitik als nationales und ethisches Postulat}

Mit dieser nüchternen, die Eigengesetzlichkeiten der kapitalistischen Marktwirtschaft und die Interessen der an ihr Beteiligten ernst nehmenden Perspektive in der Formulierungsphase von Sozialwissenschaft und Sozialpolitik kontrastiert das sozialpolitische Pathos in der Zeit des Kaiserreichs. Erst im Zusammenhang mit der Sozialversicherungsgesetzgebung der 1880er Jahre etablierte sich nämlich der Begriff, Sozialpolitik‘ in der politischen Sprache, und spätestens mit dem ,neuen Kurs' Wilhelms II. gegenüber der Arbeiterfrage wurde ,Sozialpolitik' zu einem massenhaft verwendeten Terminus, wenn nicht zu einem öffentlichen Schlagwort. Und dementsprechend vage wurde die Wortverwendung, ,scheint doch der Ausdruck Sozialpolitik noch jetzt eine besondere Anziehungskraft auf die Geister auszuüben, womit es dann zusammenhängt, daß dieser Ausdruck zur Bezeichnung derjenigen Sphäre oder Richtung der Politik verwendet wird, welche dem betreffenden Gelehrten oder Staatsmann als die wichtigste, bedeutsamste erscheint. ${ }^{.16}$ Aus dieser Situation resultierten fortgesetzte Debatten über den Begriff der Sozialpolitik, die bis gegen Ende der Weimarer Zeit dauerten. Sie verdienen unser Interesse, weil gerade in diesem Zusammenhang gemeinsinn- und gemeinwohlbezogene Diskurse an Einfluß gewannen, allerdings unter anderen Bezeichnungen.

Die Situation hatte sich durch die nationale Einigung grundlegend gewandelt. Schon 1859 hatte Heinrich von Treitschke gegen die Gesellschaftswissenschaften Front gemacht und die Unterscheidung von Staats- und Gesellschaftswissenschaften in Frage

\footnotetext{
13 Vgl. Kaufmann 1988.

14 Heimann 1980, S. $135 \mathrm{ff}$.

$15 \mathrm{Vgl}$. Abschnitt 1.4.

16 Bortkiewicz 1899, S. 348.
} 
gestellt: „Die ganze Staatswissenschaft ist sozialpolitisch; sie hat zu zeigen, wie der Gedanke der Volkseinheit sich in der Mannigfaltigkeit der Sonderbestrebungen des Volkes verwirklicht." ${ }^{17}$ Mit der Reichsgründung gewann das nationale Einheitsdenken ein Pathos, welches die gesellschaftlichen Verhältnisse eindeutig dem Staate unterordnete. Dennoch begleitete die soziale Frage als ,Arbeiterfrage" das neue Reich, wobei die politische Lösung jedoch nicht in der politischen Gleichberechtigung, sondern in einer ,sozialen Gesetzgebung' gesucht wurde, die die politischen Forderungen der Arbeiterschaft stillstellen sollte. Auch in Frankreich dominierte das nationale Denken die soziale Frage, allerdings unter anderen Vorzeichen und mit anderen Ergebnissen.

\subsubsection{Gustav von Schmoller}

Im deutschen Reich war der bei weitem einflußreichste Wirtschafts- und Sozialwissenschaftler dieser Zeit der 1908 geadelte Gustav Schmoller (1838-1917). ${ }^{18}$ Schon im Alter von 26 Jahren hatte er in den ,Preußischen Jahrbüchern' einen wegweisenden Aufsatz zur Arbeiterfrage veröffentlicht. ${ }^{19}$ Er gehörte zu den Mitbegrüindern des ,Vereins für Socialpolitik' und galt als das Haupt der jüngeren historischen Schule der deutschen Nationalökonomie. Seine Begründung der Forderung nach sozialen Reformen folgte aus einer ganz anderen Perspektive als derjenigen Lorenz von Steins, dessen Schriften seiner Generation ,chinesisch" vorkämen, wie er einmal spaßig bemerkte.

Für Schmoller wie für die meisten Mitglieder des Vereins für Socialpolitik bildete die soziale Benachteiligung der Arbeiter den Ausgangspunkt für die Forderung nach staatlichen Sozialgesetzen. Diese Forderung nach staatlichen Eingriffen stand in ausdrücklichem Widerspruch zur herrschenden ,manchesterlichen' Lehre der im ,Kongreß deutscher Volkswirte' zusammengeschlossenen Ökonomen, doch handelte es sich hier nicht nur um einen politischen, sondern auch um einen wissenschaftstheoretischen Gegensatz, wie nicht zuletzt der Methodenstreit zwischen Carl Menger und Gustav Schmoller gezeigt hat. Schmollers volkswirtschaftlicher Ansatz war institutionalistisch und nicht tauschtheoretisch, und er interpretierte die sozialen Ungleichheiten seiner Zeit im Kontext einer kulturevolutorischen Perspektive:

\footnotetext{
„Die Geschichte entrollt, wenn wir näher zusehen, vor unseren Blicken einen Stufengang von socialen Organisationsformen, von Epochen des socialen Lebens und des socialen Rechtes, von denen jede schwer mit der andern gerungen, bis sie sie verdrängt. Jede folgende streift die Spuren der Gewalt, der brutalen Herrschaft und rohen Ausbeutung, die in älterer Zeit ausschließlich geherrscht, mehr und mehr ab, kommt zu einem edleren Verhältnis der wirtschaftlichen Klassen untereinander, erkennt die Gleichberechtigung der Menschen mehr an, fordert mehr eine sittliche Wechselwirkungen der verschiedenen, betont eine Verpflichtung der höherstehenden Klassen zur Hebung der unteren; kurz jede Epoche fordert Dinge, welche früheren Jahrhunderten unbekannt und unverständlich waren. ${ }^{20}$

17 Treitschke 1859, S. 82 f.

18 Schmollers Position findet in jüngster Zeit erneute Beachtung, nachdem sie jahrzehntelang ,tot ${ }^{*}$ schien. Wichtige Arbeiten wurden neu aufgelegt in Schmoller 1998. Vgl. auch Backhaus 1993.

19 Schmoller 1864.

20 Schmoller 1998a, S. 76.
} 
Die Lösung der Arbeiterfrage war für Schmoller somit eine zugleich ethisch, politisch und sozial begründete Forderung an den preußischen Staat, zu deren Anwalt er sich machte, doch handelte es sich hierbei nicht um eine von außen an die Verhältnisse herangetragene ethische Forderung, sondern um eine dem geschichtlichen Prozeß immanente Höherentwicklung des ethischen Bewußtseins, als dessen hervorragenden Träger Schmoller das preußische Königtum seit jeher ansah und nun erneut forderte:

\begin{abstract}
„Den Gefahren der socialen Zukunft kann nur durch ein Mittel die Spitze abgebrochen werden: dadurch, daß das König- und Beamtenthum, daß diese berufensten Vertreter der Staatsgedanken, diese einzig neutralen Elemente im sozialen Klassenkampf versöhnt mit dem Gedanken des liberalen Staates, ergänzt durch die besten Elemente des Parlamentarismus, entschlossen und sicher, die Initiative zu einer großen socialen Reformgesetzgebung ergreifen und an diesem Gedanken ein oder zwei Menschenalter hindurch unverrückt festhalten. ${ }^{“ 21}$
\end{abstract}

Hier also finden wir implizit einen deutlichen Appell an den Gemeinwohlauftrag des Staates und den Gemeinsinn - zum mindesten der höheren Stände und der „besten Elemente des Parlamentarismus". Der Appell klingt allerdings reichlich voluntaristisch. Oder verstand er sich selbst als Sprachrohr des ,gesunde[n] sittliche[n] Volksgeist[es]“, dessen ,ethische Tendenz [...] Gegenbestrebungen gegen solche Auswüchse und Mißbildungen, die durch das Wirtschaftsleben entstehen oder zu entstehen drohen, hervorruft"? ${ }^{22}$ Das durch die nationale Einigung genährte Bewußtsein eines gemeinsamen Ethos führte bei Schmoller auf jeden Fall zu einem harmonistischen Weltbild, im Rahmen dessen Gemeinsinn und Gemeinwohl unschwer zueinander fanden und ökonomische Erfordernisse oder Interessengegensätze unterbelichtet blieben.

Für den Mainstream der deutschen Sozialpolitiklehre blieb in der Folge bis zum Ende des Zweiten Weltkriegs die Auffassung charakteristisch, daß Sozialpolitik eine gesellschaftsintegrative Aufgabe habe. Die zentrale Bedrohung des gesellschaftlichen $\mathrm{Zu}-$ sammenhalts wurde in diesem Zusammenhang als Klassenkonflikt diagnostiziert. Demzufolge herrschte die Auffassung, zur Sozialpolitik gehörten diejenigen Maßnahmen, welche mit der staatlichen Beeinflussung der Klassenverhältnisse zu tun haben. So wurde beispielsweise die Fürsorgepolitik nicht zur Sozialpolitik gezählt. ${ }^{23}$

\title{
1.3.2 Der französische Solidarismus
}

Es empfiehlt sich an dieser Stelle ein Seitenblick auf französische Diskurse zur Grundlegung der Sozialpolitik, da hier der Bezug zur gegenwärtigen, von der Individualisierung bestimmten Problematik expliziter ist. Die französische Revolution hatte ja alle ,corps intermédiaires‘ abgeschafft und in der ,Loi Le Chapelier' (1791) auch für die Zukunft Zusammenschlüsse auf der Basis einer gleichen Wirtschaftstätigkeit für rechtswidrig erklärt. Dennoch stellte sich natürlich auch hier die Frage nach dem sozialen Zusammenhalt der individualisierten Bürger, der zunächst im Kampfruf der ,Fraternité beschworen worden war. Im 19. Jahrhundert verdrängte der Begriff der ,Solidaritéc weitgehend denjenigen der Fraternité und wurde zum Grundbegriff des

21 Ebd., S. 95 f.

22 Schmoller 1864, S. 535.

23 Erst Achinger 1939 begründete die Einbeziehung der Fürsorge in den sozialpolitischen Diskurs. 
französischen Sozialdenkens. ${ }^{24}$ In vorliegendem Zusammenhang interessiert nur der Bezug zur Sozialpolitik, welcher vor allem mit dem Namen des Staatsmannes und Friedensnobelpreisträgers Léon Bourgeois (1851-1925) verbunden ist. ${ }^{25}$

Gegen Ende des 19. Jahrunderts wurde ,Solidarité ' zum leidenschaftlichen Kampfbegriff der laizistischen Linken: Der Begriff sollte zwischen den liberalen Ansprüchen auf Freiheit einerseits und der Notwendigkeit staatlicher Intervention andererseits vermitteln. Er hatte sich von seinen älteren juristischen und moralischen Ursprüngen weitgehend abgelöst und beinhaltete nunmehr den Appell an den aufgeklärten Egoismus des Bürgertums, nicht nur an seine Unabhängigkeit, sondern auch an seine Verflochtenheit mit dem Geschick der Arbeiterklasse zu denken und rechtzeitig einer sozialen Revolution vorzubeugen. Die Argumentation berief sich auf, wissenschaftliche Grundlagen', um die "solidarité sociale et morale" ${ }^{26}$ zu begründen. Bourgeois ging von der Annahme eines Quasi-Kontraktes aus, der die Grundlage der Rechts- und Sozialordnung eines Landes bildet. Jeder Mensch werde als Schuldner der Assoziation geboren, der er zugehörig ist, und die bereits vor seiner Geburt die Grundlagen für seine Lebensmöglichkeiten geschaffen habe. Deshalb habe er sich der Pflicht zu unterziehen, seinerseits für die weniger Begünstigten seiner Mitmenschen ,solidarisch" einzustehen. Hiervon ausgehend forderte er die kostenlose staatliche Schulbildung für jedermann, die Einführung von Sozialversicherungen und ein progressives Steuersystem. Dieses solidaristische Programm hat zwar die politische Rhetorik der wohlfahrtsstaatlichen Entwicklung in Frankreich bis heute bestimmt ${ }^{27}$ doch wurden seine Ziele durch die institutionelle $\mathrm{Ab}$ sicherung der Interessen der Wohlhabenden stets erneut unterlaufen.

Emile Durkheim durchschaute die individualistische Engführung dieser tauschtheoretischen Begründung von Solidarität und suchte sie durch eine soziologische zu ersetzen. Aber auch Bourgeois selbst appellierte nicht nur an den aufgeklärten Egoismus, sondern auch an die „moralische Idee“, ${ }^{28}$ welche die wechselseitigige Abhängigkeit der Menschen auf den Begriff bringt. Wir finden bei ihm eine ähnliche Argumentation wie bei Lorenz von Stein, allerdings auf einem geringeren analytischen Niveau. Insbesondere fehlt die Thematisierung des intermediären Bereichs ganz. Im Horizont der nationalen Idee war der Appell an den Gemeinsinn, wenn wir den Solidarismus von Bourgeois so interpretieren dürfen, ganz selbstverständlich auf den Rahmen des Nationalstaates bezogen, der auch die Kriterien des Gemeinwohls vorgab.

\subsection{Weimar: Das staatszentrierte sozialpolitische Projekt in der Krise}

Bereits während des Ersten Weltkriegs hatte die vom deutschen Bürgertum nicht erwartete nationale Solidarität der Arbeiterschaft und die kriegsbedingte Zwangsrekrutierung von Arbeitskräften eine Aufwertung der Gewerkschaften gefördert. Und mit der Grün-

24 Einen guten deutschsprachigen Überblick bietet nunmehr Zoll 2000, S. 19-89.

$25 \mathrm{Vgl}$. insb. Bourgeois 1896; sowie Hayward 1961.

26 Hatzfeld 1971, S. 272.

$27 \mathrm{Vgl}$. Bode 1999.

28 Bourgeois 1896, S.17. 
dung der ,Zentralarbeitsgemeinschaft" von Arbeitgebern und Gewerkschaften unmittelbar nach Kriegsende, der Verabschiedung der Weimarer Reichsverfassung und der Wahl des Sozialdemokraten Friedrich Ebert zum Reichspräsidenten schien die lange ersehnte politische und wirtschaftliche Gleichberechtigung der Arbeiter erreicht und die bisherige Idee der Sozialpolitik als ,Lösung der Arbeiterfrage' an ihr Ziel gekommen. Aber der politische und wirtschaftliche Niedergang der Nachkriegszeit, der seinen Tiefpunkt in der Inflation von 1922/23 fand, überforderte die wirtschafts- und sozialpolitische Kompromißfähigkeit von beiden, Unternehmerschaft und Gewerkschaften, und führte zu einer tiefen Entfremdung der Tarifparteien, die in der Folge nur noch auf der Basis staatlich vermittelter Schlichtungssprüche zu kooperieren vermochten. Diese „Krise der Sozialpolitik“29 ${ }^{\star 29}$ war zugleich eine Krise der sozialpolitischen Institutionen und der sozialwissenschaftlichen Deutungsmuster. Am deutlichsten in Frage gestellt wurde dabei das bisherige Konzept einer staatlichen Sozialpolitik.

Götz Briefs, dessen Analyse der Krisendiskussion zu den klärendsten Beiträgen der Zeit gehörte, wies auf den abstrakten, bürokratischen Charakter staatlicher Sozialpolitik hin:

„Sie hatte keinen Kontakt [...] mit der Seele des einfachen Mannes; sie wirkte von außen und ,oben': hart, kalt, generalisierend, sachlich; und fand von keiner Seite die Wärme der Anerkennung, die sie nach ihrer objektiven Leistung verdient hätte [...]. Sie kam nicht zu ihrem sinnvollen Zweck: zur sozialen Befriedung. ${ }^{\text {c30 }}$

Briefs betonte, das bisherige Nebeneinander von individualistischer Wirtschaftspolitik und staatlicher Sozialpolitik sei nicht zu halten. Vielmehr müßten „sozialpolitische Minima" in die Wirtschaft selbst eingebaut werden. Damit verwies er auf vielfältige Strömungen in der frühen Weimarer Zeit, welche eine Ordnung des Wirtschaftslebens auf der Basis von Verhandlungen zwischen Arbeitgebern und Arbeitnehmern aufbauen wollten.

Die theoretischen Grundlagen für diese, erst nach dem Zweiten Weltkrieg wirksam werdende autonome Politik der Tarifparteien hatte schon vor 1918 der sozialdemokratische Jurist Hugo Sinzheimer gelegt, der als Begründer der Lehre vom Kollektivvertrag auch als erster Theoretiker des Korporatismus gelten kann. Die analytische Leistung, welche Sinzheimer zu seinen uns heute nahezu selbstverständlichen Positionen geführt hat, wird nur deutlich vor dem Hintergrund des hoheitlichen preußischen Staatsverständnisses einerseits und der römisch-rechtlich fundierten, ausschließlich individualistisch-privatrechtlichen Auffassung des Arbeitsverhältnisses andererseits. Gegen alle syndikalistischen Versuche einer ,Rätedemokratie', aber auch gegen alle unitaristischen Vorstellungen deutscher Nationalstaatlichkeit betonte er die Differenz zwischen der politischen und der Wirtschaftsverfassung, analog zur Hegelschen Unterscheidung von ,Staat" und , bürgerlicher Gesellschaft".

„Die reine politische Demokratie kann auf die sozialen Verhältnisse nur durch Gesetz und staatliche Verwaltung einwirken. Beides aber ist unzureichend, um der sozialen Entwicklung so dienlich zu sein, wie wir es als Sozialdemokraten wünschen müssen. [...] Daraus ergibt

29 Ein geläufiger Topos der zwanziger Jahre; zur Analyse vgl. insbesondere Leubuscher 1923.

${ }^{30}$ Briefs 1923, S. 11. 
sich, daß die politische Demokratie notwendig einer Ergänzung bedarf. Die gesellschaftlichen, namentlich die wirtschaftlichen Interessen bedürfen besonderer Formen, in denen sie sich unmittelbar und selbständig auswirken können. Die politische Demokratie muß diese Formen schaffen. Sie werden geschaffen, wenn in dem Staat neben der politischen Verfassung eine eigene Wirtschaftsverfassung begründet wird, die durch die wirtschaftlichen Kräfte selbst auf dem Grunde staatlicher Grundnormen die wirtschaftlich-organisatorischen Aufgaben zur Erledigung bringt. ".31

Sinzheimer entdeckte also den intermediären Bereich neu und plädierte für eine ,soziale Selbstbestimmung im Recht" ${ }^{32}$ Zwischen die staatliche und die individualvertragliche Sphäre schiebt sich die beide vermittelnde Sphäre des ,sozialen Rechts', also institutionelle Regelungen, in denen öffentlich-rechtliche und privatrechtliche Verhältnisse im Hinblick auf die Erreichung bestimmter Zwecke zusammenwirken. Eine ähnliche Idee hat der Soziologe Georges Gurvitch (1932/1972) im Zusammenhang des französischen Institutionalismus entwickelt. Während Gurvitch die Notwendigkeit eines normativen Konsenses für die Institutionengenese betonte und - insofern ähnlich Schmoller - im Horizont einer ethosbedingten Integration verblieb, betonte Sinzheimer die Möglichkeit einer Vermittlung antagonistischer Interessen im Rahmen staatlich regulierter Verfahren. ${ }^{33}$ Eben dies geschieht heute im Arbeits- und Sozialrecht, vor allem auch im Bereich von Tarifvertragsrecht und Mitbestimmung.

Mit dem Fragwürdigwerden einer umfassenden Integrationsfunktion des Staates wurde auch die Rede von Gemeinwohl und Gemeinsinn mehrdeutig. Sobald der Glaube an einen wohlmeinenden Monarchen oder Beamtenstaat abhanden kommt, der aus inhaltlicher Einsicht (und sei es aufgrund sozialwissenschaftlicher Beratung!) das gemeine Wohl zu bestimmen vermag, kann sich der basale Konsens nur noch auf die Verfahren beziehen, im Rahmen derer die Repräsentanten unterschiedlicher Interessen um gemeinwohlverträgliche Lösungen ringen. Während im Falle staatlicher Gesetze das Ergebnis derartiger Auseinandersetzungen notfalls mit Hilfe des Gewaltmonopols des Staates durchgesetzt werden kann, bleibt eine solche Lösung im Rahmen kollektivvertraglicher Regelungen fragwürdig. Korporatistische Arrangements sind nur so lange funktionsfähig, als die interne Bindungswirkung verbandlicher Zusagen effektiv ist. , Gemeinsinn' bezieht sich hier somit nicht mehr unmittelbar auf das staatliche Ganze, sondern auf das Ganze des je eigenen Verbandes, und dasselbe gilt grundsätzlich für alle Formen freiwilliger Assoziation.

Korporatistische Arrangements, die wir in Deutschland derzeit nicht nur im Tarifvertragswesen, sondern z.B. auch im Gesundheitswesen kennen, haben den großen Vorteil, das Ausmaß an erforderlichem individuellen Gemeinsinn auf den Bereich der in der Regel ähnlich Gesinnten und ähnlich Interessierten zu reduzieren. Natürlich mag es auch hier Interessengegensätze geben, man denke an die gegenwärtigen Konflikte zwischen verschiedenen Facharztgruppen! Und es liegt dann nicht zuletzt an indirekten staatlichen Verstärkungen der Zugehörigkeitsbedingungen - konkret also der öffentlichrechtlichen Verkammerung des Arztberufs - daß die Folgebereitschaft erhalten bleibt,

31 Sinzheimer 1976 , S. $326 \mathrm{f}$.

32 Sinzheimer 1916.

33 Vgl. dazu die kritische Auseinandersetzung seitens Sinzheimers 1976, Bd. 2, S. 164-187. 
und nicht an einem besonderen Gemeinsinn. Die Integrationskraft selbstbestimmter Kollektivverträge bedarf somit in der Regel staatlicher Verstärkungen, um dauerhaft wirksam zu sein. Dies hat Sinzheimer in aller Klarheit gesehen. Das korporatistische Integrationsmodell stellt sowohl die Allzuständigkeit des Staates als auch die willkürliche Unabhängigkeit des Individuums und mit ihr der Unternehmerschaft in Frage, ermöglicht aber einen intermediären Regelungsbereich durch Interessenausgleich unter gemeinsam Betroffenen, der sich mit Bezug auf bestimmte Problemlagen als steuerungstheoretisch überlegen erweist.

\subsection{Sozialpolitik als Gestaltung von Lebenslagen}

Sowohl das aufstrebende Paradigma der ,Sozialen Marktwirtschaft ${ }^{64}$ als auch die fortgesetzte Diversifizierung der Problemlagen und die Individualisierung der Lebensorientierungen im Zuge einer allmählichen Tertiarisierung der Wirtschaft ließen nach dem Zweiten Weltkrieg die herkömmliche Vorstellung einer Dominanz gemeinsamer ,Klassen-' oder , berufsständischer Interessen' immer fragwürdiger werden.

Die Umorientierung des sozialpolitischen Denkens erfolgte vor allem durch Ludwig Preller und Gerhard Weisser. Preller definierte: „Sozialpolitik wirkt vom Aspekt des Arbeitslebens aus auf die Struktur der menschlichen Gesellschaft im Sinne des Menschen als eines Wertes eigener Prägung. "35 Damit verdeutlichte er eine doppelte, , personfunktionale ${ }^{6}$ und ,systemfunktionale' ${ }^{6}$ Referenz von Sozialpolitik. ,Sozial' ist nur eine Politik, die sowohl den individuellen Bedürfnissen der jeweils Betroffenen, als auch der kollektiven Wohlfahrt dient. Sozialpolitische Interventionen lassen sich nur rechtfertigen, soweit sie zugleich individuellen Nutzen stiften und positive externe Effekte bzw. die Reduktion negativer externer Effekte des Wirtschaftslebens erwarten lassen.

Zum theoretischen Grundbegriff dieser neuen, nicht mehr gruppen- sondern personenbezogenen Perspektive wurde der von Gerhard Weisser eingeführte Begriff der Lebenslage: „Als Lebenslage gilt der Spielraum, den die äußeren Umstände dem Menschen für die Erfüllung der Grundanliegen bieten, die er bei unbehinderter und gründlicher Selbstbesinnung für den Sinn seines Lebens ansieht. “36 Bemerkenswert an dieser Definition ist ihr breiter, über den Bereich der Ökonomie hinausweisender Zuschnitt. Es geht nicht um bloße ,Bedürfnisbefriedigung", als deren Maßstab die Einkommenshöhe gelten könnte. Es geht vielmehr um den Handlungsspielraum zur Verfolgung von ,Grundanliegen“, wobei Weisser annahm, „daß die Menschen nicht nur Interessen sinnlicher Art haben, sondern auch geistiger Interessen und Bindungen fähig sind, die, wo sie auftreten, das Handeln [...] aus sich heraus bestimmen. ${ }^{\text {“37 }}$ Sozialpolitik beeinflußt demzufolge „Chancen der Selbstverwirklichung“ und „Stellungen der Menschen im Gesellschafts- bzw. Produktionsprozeß $“{ }^{38}$ Dabei wurde mit einer gewissen

\footnotetext{
34 Dazu Blum 1969.

35 Preller 1962, S. 291.

36 Weisser 1959, S.635.

3 Ebd., 638.

38 Thiemeier 1988, S.75.
} 
Selbstverständlichkeit vorausgesetzt, daß durch eine gleichmäßigere Verteilung von Elementen der Lebenslage (z.B. Bildung, Einkommen, soziale Infrastruktur) auch wünschenswerte ,systemfunktionale" Wirkungen, also eine Förderung des ,Gemeinwohls" erzielt würde. Mit dem Verständnis von Sozialpolitik als Gestaltung von Lebenslagen verschob sich das aktivierende Zentrum wiederum in den Bereich der Politik und näherte sich damit älteren Begriffsverständnissen an.

Das Lebenslagekonzept gestattet eine realitätsnahe Konzeptualisierung des Wirkungsfeldes von Sozialpolitik, es konzeptualisiert jedoch nicht die Sozialpolitik selbst als intervenierenden Proze $\beta .{ }^{39}$ In dieser Hinsicht war Weisser noch stark von der voluntaristischen Auffassung der älteren Sozialpolitiklehre geprägt, die von einem idealisierten, einheitlichen Akteur ,Staat ${ }^{*}$ ausging, wobei es die Aufgabe der wissenschaftlichen Sozialpolitik war, sich sozusagen den Kopf dieses Akteurs zu zerbrechen und ihm rationale Ratschläge für die Erreichung der von ihm gesetzten Ziele zu erteilen. Auch Weisser unterstellte in seinem Konzept noch einen gemeinsamen Horizont von beratender Sozialwissenschaft und politischen Entscheidungsträgern und problematisierte weder den Prozeß̉ sozialpolitischer Wiilensbildung noch die Ümsetzung sozialpolitischer Entscheidungen.

Diese Auffassung wurde unter dem Einfluß soziologischer und politikwissenschaftlicher Fortschritte zunehmend fragwürdig. Gute politische Absichten, Leitbilder oder ,Zielsysteme" gewährleisten noch keine entsprechenden ökonomischen oder sozialen Wirkungen. Vielmehr sind sozialpolitische Programme sowohl hinsichtlich ihrer Entstehung als auch hinsichtlich ihrer Implementation vom Zusammenwirken zwischen gesellschaftlichen und staatlichen Akteuren abhängig. Zudem entwickeln die einmal entstandenen sozialpolitischen Einrichtungen selbst eine Eigendynamik und oftmals unvorhergesehene Nebenwirkungen. Während das ältere Nachdenken über Sozialpolitik von den Intentionen oder der „Richtung des staatlichen Eingreifens“ (v. Bortkiewicz) ausgegangen war, traten jetzt die Wirkungen staatlicher Interventionen in den Vordergrund des wissenschaftlichen Interesses. Die Kontingenz der Verhältnisse von gesellschaftlichen Kräften, Wissenschaft, Politik und Verwaltung wurde allmählich denkbar.

Ohne auf dieses, heute vielfach als Steuerungsproblematik bezeichnete Thema hier schon einzugehen, ${ }^{40}$ ergibt sich aus dieser uns bis heute in der politischen Praxis begleitenden Bestimmung von Sozialpolitik als staatlicher Einflußnahme auf die Verteilung von Lebenslagen eine neuerliche Komplizierung der Gemeinwohlbestimmung. Das Gemeinwohl wird nun vielfach mit Problemen der distributiven Gerechtigkeit in Verbindung gebracht. Es geht nicht mehr nur um die Gewährleistung bestimmter Grundrechte für jedermann, sondern um die verteilungswirksame Ausgestaltung dieser Rechte, die sich zudem immer stärker ausdifferenzieren. Besonders hart wird diese Auseinandersetzung immer dann, wenn es nicht um die Verteilung von Zuwächsen, sondern um Leistungskürzungen geht. Hiergegen ,Gemeinsinn“ mobilisieren zu wollen, ist ebenso moralisierend-naiv, wie wenn die Gefährdung von Besitzständen zu einer Gefährdung von Grundrechten aufgebauscht wird. Wie die historisch und international vergleichende Betrachtung von Sozialpolitik lehrt, können sehr unterschiedliche Vertei-

\footnotetext{
39 Hierzu Kaufmann 1982.

$40 \mathrm{Vgl}$. hierzu Abschnitt 2.3.3.
} 
lungsmuster ,gemeinwohlverträglich“ sein. Wer Verteilungsfragen ins Zentrum des Gemeinwohls rückt, höhlt dessen normativen Gehalt aus.

\section{Gemeinwohl und Gemeinsinn aus bindungssoziologischer Perspektive}

Die gegenwärtige deutsche Diskussion um, Globalisierung ' weist eine ähnliche Struktur auf wie die damalige über, Industrialisierung'. Erneut sind es technische Innovationen, welche die Produktionsverhältnisse tiefgreifend verändern, allerdings wiederum nur vermittels politischer Interventionen, die ihrerseits neue Probleme nach sich ziehen. Die Deregulierung der bis dahin nationalen Finanzmärkte - eine politische Entscheidung! drängte sich vor allem als Folge einer wachsenden digitalen Vernetzung der Welt auf und ist durchaus mit den Liberalisierungsprogrammen der Maschinenära vergleichbar. Und wie sich die Industrialisierung mit der Auflösung kleinräumiger Verkehrswirtschaften und der Etablierung nationaler, Volkswirtschaften' verband, bringen die technologischen Fortschritte im Bereich von Kommunikation und Verkehr die Notwendigkeit größerer, die nationalen Grenzen überschreitender Wirtschaftsräume mit sich. Die sich in diesem Zusammenhang intensivierende ,Standortkonkurrenz' führt zu neuen Restriktionen gerade im Bereich der Sozialpolitik, und zwar sowohl hinsichtlich des Gestaltungsspielraumes des Staates als auch hinsichtlich der Verhandlungsmacht der Gewerkschaften. Wiederum scheint sich das Recht des Stärkeren durchzusetzen, zumal die sozialpolitischen Verteilungskämpfe häufig zu Lasten der Ärmsten ausgehen. Vor allem aber bringen die gewachsenen internationalen Optionen nicht nur eine höhere Mobilität der Produktionsfaktoren, sondern z.B. auch neue Formen der Steuerverkürzung, schwer kontrollierbare Wanderungen und interkulturelle Beeinflussungen mit sich, welche den bisherigen Kontext politischer Loyalitäten in Frage stellen. Dies scheint der Kontext zu sein, in dem heute der Ruf nach ,Gemeinwohl' und ,Gemeinsinn" entsteht, der durchaus mit dem seinerzeitigen Ruf nach dem ,Sozialen`vergleichbar ist.

\subsection{Reformulierung des Problems}

Bisher wurden die Worte ,Gemeinwohl' und ,Gemeinsinn' undefiniert, jedoch nicht ohne bestimmten Sinn verwendet. Gemeinwohl' und ,Gemeinsinn“ sind klassische politikwissenschaftliche Kategorien, welche von Münkler und Fischer (1999) zur Strukturierung der Diskussion über die Bedeutung ,sozial-moralischer Ressourcen' für die Politik revitalisiert wurden. ,Gemeinwohl' bezeichnet in diesem Zusammenhang die Maxime eines auf die politische ,Gemeinschaft' bezogenen Handelns, ,Gemeinsinn* hingegen die Motivation oder Bereitschaft zur Gemeinwohlorientierung seitens derjenigen, die sich einer bestimmten,Gesellschaft' zurechnen. Das Problem ist die Mobilisierung eines über die bloße Interessenverfolgung, wie sie in ,gesellschaftlichen' Kontexten üblich ist, hinausgehenden ,Gemeinsinns" für die Zwecke des gemeinsamen Wohls aller Gesellschaftsglieder. Insoweit als dies gelingt, kann von einer politischen ,Gemeinschaft' gesprochen werden. 
In der älteren Literatur wurde das Verhältnis von Gemeinwohl und Gemeinsinn nicht problematisiert. Wie schon in der aristotelischen Polis-Lehre galt das ,gemeinsinnige Verhalten als politische Tugend und damit gleichzeitig als gemeinwohlförderlich. Auch die ethischen Begründungen von Sozialpolitik ${ }^{41}$ standen ganz selbstverständlich im Horizont des nationalstaatlichen Ethos und Pathos, welches gleichermaßen den sozialen Rahmen (,Nation') und auch die politische Instanz (,Staat') der Gemeinwohlwahrung bestimmte und somit dem ,Gemeinsinn' einen eindeutigen Solidaritätshorizont zuwies. ,Gemeinschaft' und ,Gesellschaft' erweisen sich in dieser Perspektive als weitgehend austauschbare Begriffe. Dies jedoch verwischt die Herausforderungen, welche von der funktionalen Differenzierung und dem wachsenden Umfang der Sozialzusammenhänge bis hin zur Globalisierung ausgehen, für die sich seit dem 19. Jahrhundert die Bezeichnung ,Gesellschaft' eingebürgert hat, mit recht unterschiedlichen Auslegungen des Begriffs im einzelnen. Es ist die Aufgabe dieses zweiten Abschnitts, mittels begrifflicher Klärungen auch die unterschiedlichen sozialen Sachverhalte zu verdeutlichen, auf die sich die politische Gemeinsinns- und Gemeinwohlrhetorik heute bezieht.

Beides, der soziale Rahmen wie auch die Instanz der Gemeinwohlwahrung werden gegenwärtig durch die gemeinhin als ,Globalisierung' bezeichneten Veränderungen fragwürdig. Zwar deckt dieser Begriff recht unterschiedliche Entwicklungen, doch konvergieren diese in der Infragestellung von Souveränität und Autonomie des Nationalstaates und damit auch des herkömmlichen Gesellschaftsbegriffs. ${ }^{42}$ Der Nationalstaat verliert seinen Charakter als umfassende ,Schicksalsgemeinschaft" und wird zunehmend zu einer politischen ,Steuerungsebene' unter anderen. Das gilt insbesondere im Horizont der EU, welcher zunehmend Rechte übertragen werden, die herkömmlicherweise als Attribute staatlicher Souveränität galten. Zugleich bewirkt die Offenheit der Grenzen für das Aus- und Einströmen von Gütern, Kapitalien, Wissensbeständen und Menschen - und damit der Relevanzgewinn transnationaler Entwicklungen, daß die Autonomie der nationalen Ebene entscheidend geschwächt wird. Kollektive Ereignisse und Lebensschicksale lassen sich nun immer weniger plausibel allein auf nationale Politik zurechnen, wie aktuellerweise die BSE-Krise zeigt. Die kürzliche Schlagzeile der BildZeitung: „Kanzler - Was sollen wir noch essen?“ macht gleichermaßen die herkömmlichen Erwartungen als auch - in ihrer Lächerlichkeit - die neue Sachlage deutlich.

Mit der Schwächung der nationalen Identifikationsebene treten zugleich auch subnationale Solidaritätshorizonte wieder stärker ins Bewußtsein, regionale und lokale Identitäten artikulieren sich erneut. Zugleich profiliert sich die Europäische Union als neuer Träger von Gemeinwohlfunktionen, ganz abgesehen von der diskursiven Aktualisierung eines „Weltgemeinwohls", für das eine politische Trägerstruktur noch kaum in Sicht ist. Es ist diese Vervielfältigung der Solidaritätshorizonte, welche m.E. bewirkt, daß heute „Gemeinsinn als motivationale Voraussetzung jedweder Gemeinwohlorientierung eine äußerst knappe soziomoralische Ressource darstellt. ${ }^{43}$ Die anschließende These von Münkler und Fischer, daß der Verbrauch sozialmoralischer Ressourcen „erheblich steigen (dürfte), wenn die Größe jenes politisch-sozialen Ge-

41 $\mathrm{Vgl}$. Abschnitt 1.3.

42 Vgl. Kaufmann 1998.

43 Münkler/Fischer 1999, S. 238. 
meinwesens, das die Zielgruppe von Wohlfahrtsstreben bildet, definitorisch ausgeweitet wird“" ${ }^{44}$ trifft dagegen das Problem nur peripher. Das Größenwachstum politisch konstituierter Sozialzusammenhänge bedarf zu seiner Stabilisierung einer Binnenstrukturierung, deren Funktionsweise von der unmittelbaren Wirkung sozialmoralischer Ressourcen weitgehend unabhängig geworden ist. Damit löst sich der herkömmliche Zusammenhang von Gemeinwohl und Gemeinsinn auf. Dennoch werden sozialmoralische Ressourcen nicht überflüssig, aber ihre Mobilisierbarkeit wird, wie zu zeigen sein wird, in hohem Maße kontextabhängig.

Verständlicherweise sprechen Münkler und Fischer wiederholt von ,politischer Gemeinschaft", wenn sie auf den Zusammenhang von Gemeinsinn und Gemeinwohl rekurrieren. Denn aus soziologischer Sicht erscheint dieser Zusammenhang nur unter den Bedingungen enger Vergemeinschaftung relativ unproblematisch. In welchem Sinne läßt sich aber der verselbständigte moderne Staat noch als Gemeinschaft begreifen? Das begriffsprägende Leitbild der aristotelischen Theorie der Polis hat ja seine Tragfähigkeit im Zuge der modernisierenden Differenzierungsprozesse verloren, wie bereits die Ausführungen des ersten Teils zeigen sollten. Die bürgerliche Gesellschaft im Hegelschen Sinne kennt keine gemeinschaftlichen Beziehungen; zum Ort des gemeinschaftlichen Ethos der „natürlichen Sittlichkeit" wurde bei Hegel die Familie. Der Staat dagegen beruht auf dem neuen Bindungsmedium des positivierten Rechts. Diese Grundgedanken sind in neueren soziologischen Gesellschaftstheorien, insbesondere bei Niklas Luhmann und Talcott Parsons, in problemaufschließender Weise ausgearbeitet worden. Dabei wurde gegenüber Hegel nicht nur das Differenzierungsdenken radikalisiert, sondern vor allem auch der parallel zur Ausdifferenzierung gesellschaftlicher Teilsysteme sich vollziehenden Verselbständigung unterschiedlicher Ebenen sozialer Organisation Beachtung geschenkt. Auf dieses Phänomen sind wir oben bereits im Zusammenhang mit der Entstehung korporatistischer Arrangements ${ }^{45}$ gestoßen. Als Konsequenz der damit angedeuteten Steigerung gesellschaftlicher Komplexität ordnen sich die mit den Begriffen ,Gemeinwohl' und ,Gemeinsinn' angedeuteten Problembezüge neu. Wir müssen von einer Gesellschaftsvorstellung des ,integrierten Ganzen' Abschied nehmen und ,Gesellschaft" als differenzierten, durch Macht, Austausch und unterschiedliche Formen sozialer Bindung konstituierten Gesamtzusammenhang zu begreifen suchen.

\subsection{Konstitutionalisierung als Bezugsproblem von Gemeinwohl}

Mit besonderer Radikalität hat Niklas Luhmann den aus der aristotelischen Politiklehre stammenden Zusammenhang zwischen Bürgertugend und Politik in Frage gestellt. Sein Begriff des politischen Systems als ,autopoietisches System der Machtkonstitution und -benutzung ${ }^{46}$ verweist individuelle Motivationen in den Bereich der personalen Systemumwelt, von der das System nur selektiven Gebrauch zu seinen eigenen Zwecken macht. Bürgertugend - ,Gemeinsinn ${ }^{6}$ - ist dazu bestimmt, politisch instrumentalisiert zu

44 Ebd.

45 Abschnitt 1.4

46 Luhmann 2000, S. 28. 
werden und hat keinerlei eigenständiges Gewicht. Ähnlich und noch radikaler geht Luhmann mit der Moral um: „Empirisch gesehen ist moralische Kommunikation nahe am Streit und damit in der Nähe von Gewalt angesiedelt. Sie führt im Ausdruck von Achtung und Mißachtung zu einem Überengagement der Beteiligten." ${ }^{\text {47 }}$ Im Gegensatz zu Durkheim und vielen Konsenstheorien erscheint Moral hier somit nicht als Moment sozialer Bindung, sondern als Moment sozialer Trenmung. Der gesellschaftliche Zusammenhang konstituiert sich nach Luhmann durch jede Form der Kommunikation und gewinnt dadurch einen hochgradig kontingenten Charakter. Die notwendige Erwartbarkeit von Anschlußkommunikationen kommt durch die teilsystemspezifische Codierung der jeweiligen Kommunikationsformen zustande. Somit orientiert sich sozial erwartbare Kommunikation an den institutionalisierten ,Codes' des jeweiligen Teilsystems. Zum Beispiel: Nur wer sich auf den Code Macht einläßt, hat die Chance, politisch Gehör zu finden; und nur wer über Geld verfügt, kann wirtschaftlich etwas bewegen. Wer jedoch nicht kommuniziert (oder nicht in teilsystemisch bestimmter Form kommunizieren kann!), schließt sich von Gesellschaft bzw. den jeweiligen Teilsystemen aus. Wohlfahrtsstaatliche Politik findet deshalb ihre spezifische Legitimation in der Gewährleistung von Teilhabechancen an allen relevanten Funktionssystemen einer Gesellschaft (Inklusion).

Dem Gemeinwohlbegriff spricht Luhmann die Rolle einer Legitimation erzeugenden „Kontingenzformel“ zu, deren Wirkung jedoch historisch verbraucht erscheint und sich weniger als der ihn historisch ersetzende Begriff der Legitimität eignet, um die mit der Ausdifferenzierung des politischen Systems einhergehenden Paradoxien zu entfalten. ${ }^{48}$

Luhmanns Moralbegriff entspricht allerdings nicht demjenigen der praktischen Philosophie, sondern orientiert sich weitgehend am Vulgärgebrauch. Otfried Höffe hat in kritischer Auseinandersetzung mit Luhmann „drei Funktionen einer politischen Moral“ herausgearbeitet, welche diese auf unterschiedlichen Ebenen sozialer Emergenz entfaltet: Auf der Ebene des Gemeinwesens bzw. des politischen Teilsystems wirkt Moral politiklegitimierend, auf der Ebene der politischen Regeln und Institutionen wirkt Moral politiknormierend, und auf der Ebene der an Politik beteiligten Personen instruiert Moral das politische Urteil. ${ }^{49}$ Hier allerdings geht es bei Moral nicht um die konkret im Rahmen eines bestimmten Ethos vorfindlichen sozialen Normen, sondern um die ethische Reflexion der Bedingungen allgemein zustimmungsfähiger Normen, welche vielfach auch als, gesellschaftliche Werte' bezeichnet werden. Dementsprechend wird bei Höffe das Gemeinwohl nicht demokratietheoretisch als Resultante politischer Auseinandersetzungen bestimmt, sondern nur, was allgemein zustimmungsfähig ist, verdient als gemeinwohldienlich bezeichnet zu werden. ${ }^{50}$ Dabei handelt es sich im wesentlichen

${ }^{47}$ Luhmann 1990, S. 26.

$48 \mathrm{Vgl}$. Luhmann 2000, S. $120 \mathrm{ff}$.

49 Vgl. Höffe 1991, S. 305 ff.

${ }^{50}$ Das ist ein erkennbar restriktiver Begriff des Gemeinwohls, der jedoch den Vorteil weitgehender Eindeutigkeit besitzt. Die Alternative besteht entweder in einer inhaltlichen Entleerung des Begriffs, indem jedes Resultat politischer Auseinandersetzungen unter demokratischen Bedingungen als Gemeinwohl anerkannt wird. Oder aber der Gemeinwohlbegriff wird von der Ebene der größtmöglichen gesellschaftlichen Reichweite gelöst und mit partikulären Prozessen der Konsensbildung 
um das Prinzip der Volkssouveränität auf der Ebene der Konstitution politischer Systeme sowie um die Prinzipien der Menschenrechte, der Gewaltenteilung und der Rechtsstaatlichkeit auf der Ebene der Institutionalisierung politischer Herrschaft. Diese Kriterien der Moralität von Politik erscheinen jedoch im Sinne eines herkömmlichen, am Handeln von Personen orientierten Sinne als „entmoralisiert“. Ihre Wirksamkeit liegt im wesentlichen auf der konstitutionellen Ebene des Gemeinwesens und ist von den Gesinnungen der Bürger ,tatsächlich [...] unabhängig“. ${ }^{51}$ Demzufolge erscheint in modernen Demokratien das Gemeinwohl inhaltlich durch die Kernbestimmungen der Verfassung gewährleistet und gleichzeitig auf sie beschränkt. Diese Überlegung ist auch mit der frühen These von Luhmann (1965/1999) vereinbar, daß die Institutionalisierung von Grundrechten die Bedingung funktionaler Vergesellschaftung und damit der Verunmöglichung des herkömmlichen Gemeinwohldiskurses ist.

Nach Höffe spielen die moralischen Gesinnungen dagegen auf der dritten Ebene der „sittlich-politischen Urteilskraft“ eine konstitutive Rolle. Als moralische Kompetenzen erscheinen hier ,moralische Sensibilität“ und die „Fähigkeit, universalistische Prinzipien [...] situationstypengerecht zu applizieren." Die sittlich-politische Urteilskraft beruht jedoch nicht nur auf moralischen, sondern gleichermaßen auf kognitiven Kompetenzen, ohne die sie ihre praktischen Aufgaben der Güterabwägung, der Setzung von Prioritäten oder des Treffens richtiger Zeitpunkte zum Handeln nicht erfüllen könnte. ${ }^{52}$ Offensichtlich kommt politische Urteilskraft um so eher zum Tragen, je stärker eine Person in politische Verantwortungszusammenhänge eingebunden ist. Von ,Gemein$\operatorname{sinn}^{*}$ als einer mobilisierbaren sozialmoralischen Ressource ist auch hier nicht die Rede. Vielmehr erweist sich das Gemeinwohl als Inbegriff allgemein zustimmungsfähiger Prinzipien von einem spezifischen ,Gemeinsinn` als unabhängig.

\subsection{Solidarität als Bezugsproblem von Gemeinsinn}

\subsubsection{Vergemeinschaftung und Vergesellschaftung}

Versuche der Mobilisierung sozialmoralischer Ressourcen bedienten sich in Deutschland vornehmlich der Semantik von ,Gemeinschaft', ein Begriff, der wie ,Gesellschaft ${ }^{*}$ auf das Ganze des Sozialzusammenhangs zu verweisen scheint, beispielsweise in der in Verruf geratenen Version der, Volksgemeinschaft'. Wie die vielfältige Rezeption des für die Unterscheidung grundlegenden Werkes von Ferdinand Tönnies (1970) zeigt, erweist sich die Substantivierung beider Begriffe als mißverständlich, da sie einen kon-

in Beziehung gesetzt. $\mathrm{Zu}$ unterschiedlichen theoretischen Diskursen über, Gemeinwohl` vgl. Koslowski 1999.

51 Höffe 1991, S. 309. Allerdings dürfte - dies sei ergänzend hervorgehoben - die Loyalität der Bürger hinsichtlich der Einhaltung positiver Rechtsnormen, welche ja im Regelfalle keineswegs dem ethischen Kriterium allgemeiner Zustimmungsfähigkeit genügen, durchaus vom „Legitimitätsglauben“ (Max Weber) der Rechtsunterworfenen abhängen, der in diesen Kriterien immerhin seine rationale Begründung finden kann; vgl. Abschnitt 2.3.2.

52 Vgl. Höffe 1991, S. 314. In ähnlichem Sinne habe ich versucht, den Begriff der, Verantwortlichkeit' zu entwickeln, vgl. Kaufmann 1992, S. 75 ff. 
kurrierenden Gebrauch zweier totalisierender Begriffe nahelegt. „Was ist der Oberbegriff von Gemeinschaft und Gesellschaft?" fragt Georg Vobruba ${ }^{53}$ zu Recht, löst das Problem jedoch durch die Auflösung der Spannung mittels einer Überordnung des Gesellschafts- über den Gemeinschaftsbegriff. Max Weber hat - ausgehend von der Tönniesschen Unterscheidung - hilfreichere Kategorien geschaffen, welche eine differenzierte Verwendung der Dichotomie ermöglichen:

"Nergemeinschaftung“ soll eine soziale Beziehung heißen, wenn und soweit die Einstellung des sozialen Handelns [...] auf subjektiv gefühlter (affektueller oder traditionaler) Zusammengehörigkeit der Beteiligten beruht. ,Vergesellschaftung' soll eine soziale Beziehung heißen, wenn und soweit die Einstellung des sozialen Handelns auf rational (wert- oder zweckrational) motiviertem Interessenausgleich oder auf ebenso motivierter Interessenverbindung beruht. Vergesellschaftung kann typisch insbesondere (aber nicht: nur) auf rationaler Vereinbarung durch gegenseitige Zusage beruhen. Dann wird das vergesellschaftete Handeln im Rationalitätsfall orientiert a) wertrational an dem Glauben an die eigene Verbindlichkeit, - b) zweckrational an der Erwartung der Loyalität des Partners. [...] Die große Mehrzahl sozialer Beziehungen hat teils den Charakter der Vergemeinschaftuing, teils den der Vergesellschaftung. ".54

Bezogen auf die Frage nach der Rolle von Gemeinsinn und Gemeinwohl im Hinblick auf den Charakter des modernen Staates würde das Problem verfehlt, wollte man Gemeinsinn ohne das Merkmal ,gefühlter Zusammengehörigkeit der Beteiligten“ definieren. Allerdings impliziert der politikwissenschaftliche Begriff des Gemeinsinns nicht nur ein Zusammengehörigkeitsgefühl, sondern auch eine subjektive Verpflichtung zu einem Handeln im gemeinsamen Interesse, sofern ,das gemeine Wohl' es erfordert. Der Bezugsraum des gemeinen Wohls wird dabei durch die Referenzgröße des Zusammengehörigkeitsgefühls bestimmt. Im Begriff der ,politischen Gemeinschaft' ist vorausgesetzt, daß diese Referenzgröße mit dem jeweiligen politischen Gemeinwesen übereinstimme. Das ist eine unscharfe Bestimmung, denn natürlich hat ein moderner Staat „teils den Charakter der Vergemeinschaftung, teils den der Vergesellschaftung" (Weber). Diese Umschreibung bleibt jedoch in unserem Zusammenhang noch zu vage.

Talcott Parsons hat in seiner Gesellschaftstheorie mit Hilfe des Begriffs der ,gesellschaftlichen Gemeinschaft" (societal community) eine präzisere Zuordnung versucht. ${ }^{55}$ Parsons postuliert auch für moderne, großräumige, staatsförmige Gesellschaften die Zentralität eines Loyalität ${ }^{56}$ erzeugenden, durch kulturelle Werte legitimierten Normensystems, welches eine kohärente kollektive Organisation hervorbringt. Konstitutiv ist

53 Vobruba 1994, S. 17.

54 Weber 1964, S. 29 f.

5s Im Rahmen seines sog. AGIL-Schemas postulierte Parsons, daß jedes soziale System genau vier Funktionsprobleme zu lösen habe, nämlich Anpassung, Zielverwirklichung, Normenerhaltung und Integration, und daß sich zur Lösung jedes dieser Funktionsprobleme analytisch ein Subsystem von Gesellschaft identifizieren lasse. Dabei wies er der, gesellschaftlichen Gemeinschaft das Funktionsproblem der Integration zu, das im wesentlichen über die Definition von Mitgliedschaftsbedingungen gelöst wird; vgl. insb. Parsons 1972, S. 20 ff.

56 „Loyalität ist die Bereitschaft, auf angemessen, gerechtfertigte ${ }^{c}$ Appelle im Namen des Kollektivs oder des ,öffentlichen' Interesses oder Bedarfs zu reagieren." (Parsons, 1972, S. 22) Diese Umschreibung ähnelt sehr dem postulierten Strukturzusammenhang von Gemeinsinn und Gemeinwohl. 
hierfür in demokratischen Staaten eine Bürgerrolle, die als basale Form der Mitgliedschaft auf der Verfassungsebene definiert und grundsätzlich allgemein zugänglich ist. Durch die mit der Bürgerrolle verbundenen Rechte und Pflichten wird der Einzelne in den staatlichen Zusammenhang einbezogen, und diese ,Inklusion' stellt die Basis gesellschaftlicher Integration dar. Im Anschluß an Marshall (1992) können wir zwischen bürgerlichen, politischen und sozialen Rechten unterscheiden, wobei sich liberalkapitalistische und wohlfahrtsstaatliche Regime danach unterscheiden, inwieweit die sozialen Rechte als Merkmal politischer Inklusion mit anerkannt werden. Für diese primäre Ebene normativer Integration von Gesellschaft gilt das in Abschnitt 2.2 Gesagte.

Integration wird darüber hinaus durch „ein komplexes Netz sich gegenseitig durchdringender Gesamtheiten und kollektiver Loyalitäten“ hergestellt, im Rahmen eines „System(s), das durch funktionale Differenzierung und Segmentierung gekennzeichnet wird. " ${ }^{57}$ Parsons deutet hier eine komplexe Vernetzung gemeinschaftlicher und gesellschaftlicher Integrationsformen an. Zum einen haben die Gesellschaftsmitglieder in vielfältigen Rollen teil an ausdifferenzierten und segmentierten Handlungszusammenhängen, die zunehmend seibst den Charakter von Organisationen, d.h. von auf Mitgliedschaft beruhenden Handlungszusammenhängen mit eigener Akteursqualität annehmen. Das Verhältnis zwischen diesen kollektiven Akteuren ist in hohem Maße durch rationale Interessenverfolgung zu kennzeichnen, also ,gesellschaftlicher' Art. Ihre Beziehungen sind i.d.R. rechtlich und nicht moralisch normiert, und nichts kann der eigennützigen Interessenverfolgung Einhalt gebieten als zwingendes (,öffentliches') oder vereinbartes (,privates") Recht. Der formalisierte Charakter dieser Beziehungen macht sie in besonderem Maße situationsunabhängig und damit abstrakt-verläßlich. ${ }^{58} \mathrm{Die}$ individuellen Akteure dagegen sind in doppelter Weise gesellschaftlich integriert, über die Staatsbürgerrolle und ihre vielfältigen Mitgliedschaften in Organisationen. ${ }^{59}$ Die offene Frage bezieht sich hier erneut auf das Verhältnis von ,Vergemeinschaftung * und ,Vergesellschaftung' im Weberschen Sinne. Inwieweit reicht eine bloße Verbindung aus Interesse, und inwiefern setzt das Funktionieren von Organisationen als kollektiven Akteuren eine subjektiv gefühlte Zusammengehörigkeit der Beteiligten voraus?

Ein Gefühl der Zusammengehörigkeit braucht nicht durch persönliche Bekanntschaft bestimmt zu sein, es kann sich beispielsweise auch aus räumlicher oder sozialer Nähe, aus gleicher Herkunft oder aus einem gemeinsamen Schicksal ergeben. Diese situativen Momente reichen jedoch für sich allein genommen nicht aus. Es müssen normative Orientierungen hinzukommen, welche unter den Beteiligten die Anerkennung als ,Meinesgleichen' sichern. Soweit die Zusammengehörigkeit nicht personell, sondern kategorial bestimmt wird, ist sie notwendigerweise symbolisch vermittelt. ${ }^{60}$ Nicht jede gemeinsame kategoriale Zuordnung, sondern nur die durch emotional aufgeladene normative Symbole vermittelte, gemeinsame Zuordnung, oder aber eine aktuelle, als ge-

57 Parsons 1972, S. 23.

$58 \mathrm{Vgl}$. Geser 1990.

59 Daneben sind die Individuen in unterschiedlichem Ausmaße auch informell vernetzt, z.B. im Rahmen von Haushalten, Verwandtschafts- und Bekanntschaftsbeziehungen.

${ }^{60}$ Nach dem Gesagten dürfte klar sein, daß in demokratischen Staaten die Verfassung selbst eine der stärksten symbolischen Vermittlungen darstellt. 
meinsames (erfreuliches oder meist eher schmerzhaftes) Schicksal erfahrene Situation, vermag ein Gefühl der Zusammengehörigkeit zu erzeugen. Zum Begriff der Gemeinschaft gehört die grundsätzlich mobilisierbare Emotionalität der Zusammengehörigkeit, welche jedoch nur unter zusätzlichen Bedingungen als Movens eines gemeinschaftsbezogenen Handelns erscheint.

\subsubsection{Solidarität als Handlungstypus und die unterschiedlichen Sinnformen von Solidarität}

Zur präziseren Fassung des Problems führen wir im folgenden (2.3.3) ein Konzept von Solidarität als Steuerungsform ein, müssen jedoch zunächst den gemeinten Begriff von Solidarität klären. ${ }^{61}$ Während der Gemeinschaftsbegriff das Gefühl der Zusammengehörigkeit betont, wird im Solidaritätsbegriff deutlicher auf die Gemeinsamkeit des Handelns $^{62}$ abgehoben. Zwar finden sich auch viele Umschreibungen, die ,Gemeinschaft ${ }^{6}$ und ,Solidarität' in einen engen Verweisungszusammenhang bringen, aber damit wird das Spezifikum von Solidarität, insbesondere von moderner Solidarität ${ }^{63}$ verfehlt. $^{64}$ Auch für traditional fundierte Gemeinschaften gilt im übrigen, daß sie keineswegs sich dauerhaft solidarisch fühlen oder handeln; die Sozialgeschichte berichtet nicht wenig von Streit, dauerhaften Zerwürfnissen und internen Spaltungen. Auch dort bedarf es spezifischer Anlässe (z.B. ritueller Feste, kollektiver Bedrohungen oder freudiger Ereignisse), um Solidarität zu aktivieren. Für moderne Erscheinungsformen von Solidarität gilt in noch weit stärkerem Maße, daß sie kontextuell gebunden und nicht notwendigerweise auf einen konstanten Personenkreis bezogen ist. Das wurde in Abschnitt 2.1 mit dem Hinweis auf die Vervielfältigung der Solidaritätshorizonte bereits angedeutet.

Solidarität manifestiert sich stets im Handeln, nicht in bloßen Gefühlsäußerungen. Wie aber unterscheidet sich solidarisches Handeln von anderen Handlungstypen? Folgte man Max Webers Unterscheidung von Vergemeinschaftung und Vergesellschaftung, so hieße die Differenz Motivation („Einstellung des sozialen Handelns“) durch wert- oder zweckrationale Interessen einerseits oder durch Zusammengehörigkeitsgefühl andererseits. Diese Dichotomisierung orientiert sich an den vier grundlegenden Handlungstypen, ${ }^{65}$ von denen zweck- und wertrational motiviertes Handeln der Vergesellschaftung, traditional und emotional motiviertes Handeln dagegen der Vergemeinschaftung zuge-

${ }^{61}$ Vgl. zuerst Kaufmann 1984 und Hegner 1985/1991; zur Diskussion Gabriel u.a. 1997. Im folgenden wird eine vertiefende Klärung dieses Entwurfs im Lichte der aktuellen Diskussionen um ,Solidarität" angestrebt.

$62 \mathrm{Vgl}$. Zoll 2000, S. $13 \mathrm{ff}$.

63 Hierzu Gabriel/Herlth/Strohmeier 1997.

64 Ich distanziere mich deshalb auch von der These von Bayertz 1998, S. 11: „Der gemeinsame deskriptive Kern dieser heterogenen Verwendungsweisen des Begriffs [der Solidarität, F.-X.K.] besteht in der Idee eines wechselseitigen Zusammenhangs zwischen den Mitgliedern einer Gruppe von Menschen." Zwar ist die Entstehung von Solidarität im Rahmen konstituierter Sozialzusammenhänge wahrscheinlicher als in unstrukturierten Situationen, kommt aber auch dort durchaus vor, beispielsweise bei Naturkatastrophen. Vor allem ist die Umschreibung von Bayertz so weit, daß nahezu alle nicht hierarchischen Sozialformen darunter fallen.

${ }^{65} \mathrm{Vgl}$. Weber 1964, S. $17 \mathrm{f}$. 
ordnet werden. Solidarität resultiert jedoch gerade unter zeitgenössischen Bedingungen häufig aus einer wertrationalen Motivation. Man könnte auch postulieren: Wertrationalität substituiert die schwindende Traditionalität als Sinnkontext für großräumigere Formen von Solidarität. In kleinräumigen Kontexten dagegen - unter zeitgenössischen Bedingungen beispielsweise in Führungszirkeln, Clubs oder selbst permanent tagenden Verhandlungsgremien -, lassen sich durchaus Prozesse der Traditionsbildung erkennen, welche als Sinnkontext für Solidarisierungen fungieren können. ${ }^{66}$ Webers Unterscheidungen sind also zur Präzisierung des Problems nicht hilfreich.

Charakteristisch für solidarisches Handeln ist die Nachrangigkeit des Eigeninteresses bzw. das Fehlen einer egoistischen Interessenverfolgung im Handlungsvollzug. ${ }^{67}$ Solidarisches Handeln kann zwar durchaus auch im längerfristigen oder ,aufgeklärten' Eigeninteresse sein; als solidarisches wird es aber erkennbar und zurechenbar, insofern eine zweckrationale Rekonstruktion der Handlungssituation ein egoistischeres Handeln als das beobachtete nahelegen würde. ${ }^{68}$ Wir können solidarisches Handeln daher auch als kooperatives Handeln im spieltheoretischen Sinne oder als Verzicht auf die Verfolgung des eigenen Vorteils zu Lasten Dritter (free-ridership) kennzeichnen. ${ }^{69}$ Die spezifische Differenz des Begriffes wird hier also nicht wie bei Weber in den Unterschieden der Motivation, sondern in beobachtbaren Merkmalen des Handelns lokalisiert. ${ }^{70}$

In der Literatur behandelte, typische Erscheinungsformen von so definierter Solidarität lassen sich wie folgt klassifizieren:

1. Loyalität: Hier geht es im Kern um die Anerkennung bestehender Ordnungen und die Erfüllung von Regeln und berechtigten Erwartungen Dritter in Situationen, in denen mit

${ }^{66}$ Berger/Luckmann 1969, S. 72 ff. sprechen in diesem Zusammenhang von „Sedimentbildung [...]: die Erfahrung erstartt zur Erinnerung und wird zu einer erkennbaren und erinnerbaren Entität." Auch Luhmann betont für ,einfache Sozialsysteme“, daß „die Ausdifferenzierung einer eigenen Geschichte [...] ein wesentliches Moment der Ausdifferenzierung des Systems selbst" ist; Ders. 1972, S. 57.

${ }^{67}$ Hegner 1997 unterscheidet im Anschluß an Schopenhauer zwischen engstirnigem ,Egoismus` und kluger Verfolgung des ,Eigennutzes' und weist auf Fallstricke, altruistischer' Verhaltensstrategien hin. Dabei wird aus der Perspektive der Handelnden argumentiert. Demgegenüber wird hier aus der Perspektive des soziologischen Beobachters argumentiert, der deutlicher zwischen der Handlungsebene und der institutionellen Ebene der Koordination von Handlungen unterscheidet.

${ }^{68}$ Für den wichtigen Fall ,gemeinschaftlicher' (hier: ,kollektivitätsorientierter') Handlungsweisen diagnostiziert Wiesenthal 2000, S. 54 sogar ein „Verschwimmen“ der „Differenz zwischen Individual- und Kollektivgütern".

${ }^{69} \mathrm{Vgl}$. Gretschmann 1985/1991.

${ }^{70}$ Weber unterscheidet Handlungstypen nach ihrem „gemeinten Sinn“. Dabei wird „Verstehen“, d.h. eine Kongruenz von Beobachterperspektive und Handlungsperspektive vorausgesetzt, die in neueren Theorieansätzen problematisiert wird. Insbesondere die Luhmannsche Systemtheorie insistiert auf der sozialen Intransparenz, psychischer Systeme". "Gemeinter Sinn" kann sich dann nur noch auf die sozialen Kommunikationen beziehen, mit denen bestimmte beobachtbare Handlungsweisen hinsichtlich ihrer Motivation gedeutet werden. In diesem Sinne ist die folgende Typologie auch als Taxonomie verbreiteter Deutungsmuster zu verstehen. Dabei ist erneut an den ,gemischten Charakter der meisten beobachtbaren Sozialphänomene zu erinnern. Wir arbeiten hier an analytischen Unterscheidungen. 
Sanktionen für Regelverletzungen oder Enttäuschungen nicht zu rechnen ist. Das ist eine für moderne, tendenziell anonyme Gesellschaften charakteristische Umschreibung. ${ }^{71}$ Verwandte Themen sind etwa ,Legitimitätsglaube' (Max Weber), ,nicht vertragliche Elemente des Vertrags' (Durkheim/Parsons), oder, Moralität' ${ }^{72}$ Loyalität ist eine unverzichtbare Voraussetzung für die Entstehung von Vertrauen (trust) und insbesondere auch von ,Systemvertrauen", ${ }^{73}$ denn wo die systemkonstituierenden Regeln verbreitet nicht eingehalten werden, schwindet nicht nur das Vertrauen, sondern es leidet auch die effektive Operationsweise eines Systems. Zudem erhöht das Fehlen ausreichender Loyalität (z.B. ,Dienst nach Vorschrift', Korruption) die Kontrollerfordernisse. Aus ökonomischer Perspektive bewirkt Loyalität bzw. Vertrauen eine Reduktion der Transaktionskosten, und zwar sowohl in hierarchischen wie in Marktbeziehungen. Und solidarische Steuerungsformen sind geradezu konstitutiv auf Loyalität angewiesen (s.u.). Loyalität liegt somit erkennbar im objektiven Interesse aller Beteiligten; und daher ebenso die möglichste Transparenz von Regelverletzungen. Dem dient in erster Linie die Formalisierung von Regeln, sei es in der Form von Gesetzen, Verträgen oder Organisationsordnungen, und die Errichtung von Sanktionsinstanzen. ${ }^{74}$ Die Komplexität moderner Sozialverhältnisse verunmöglicht jedoch die Formalisierung aller relevanten Gesichtspunkte und ist auf das ,verantwortliche“ Handeln der Beteiligten angewiesen, also auf eine situations- und sachgerechte Handhabung von Entscheidungsspielräumen „nach Treu und Glauben". ${ }^{75}$ Loyales Handeln im hier verstandenen Sinne überschreitet somit nicht die Ebene des auch rechtlich geforderten Niveaus der Moralität, entspricht diesen Forderungen aber unabhängig von der Wahrscheinlichkeit ihrer Sanktionierung.

2. Altruismus: Zahlreiche soziale Verhaltensweisen erscheinen aus der Perspektive eigennützigen Rationalverhaltens als, irrational' bzw. als, altruistisch`. Deshalb empfiehlt sich die eingeführte Bezeichnung Altruismus. Aus der Perspektive eines erweiterten Interessebegriffs können sie vielfach aber durchaus als im eigenen Interesse liegend interpretiert werden. ${ }^{76}$ Genau genommen handelt es sich bei ,Egoismus' und ,Altruismus' stets um Zuschreibungen eines Beobachters auf nicht unmittelbar erkennbare Motive eines Handelnden, wobei als sichtbares Zeichen altruistischen Handelns wertrationale Begründungen sowie das Fehlen reziproker Handlungen des Handlungsadressaten gelten können. Im Sinne des konventionell-ökonomischen Begriffs eigennützigen Handelns - und er stellt die Kontrastfolie nahezu aller Solidaritätsdiskurse dar - können als zeitgenössisch typische altruistische Handlungsweisen etwa gelten: Wohltätigkeit, das Spenden von Blut, die Erziehung von Kindern, aber auch advokatorische Sozialbewegungen wie z.B. Tierschutz-, Umwelt- oder Dritte-Welt-Gruppen.

71 Vgl. Hirschman 1970. ,Loyalität " wird hier also in einem engeren Sinne verwendet als bei Parsons, vgl. Fn. 56.

72 Siehe z.B. Phelps 1975.

73 Zuerst Luhmann 1968.

${ }_{75}$ Dies entspricht der im folgenden (2.2.3) unterschiedenen Steuerungsform der ,Hierarchie'.

75 Vgl. Kaufmann 1992, S. 75 ff.

${ }^{76}$ Entsprechende Erklärungsmuster gehen zumeist von Adam Smiths „Theorie der ethischen Gefühle“ aus (Smith 1977). In moderner Form können wir dann z.B. von einem ,Bedürfnis nach Anerkennung* oder ,Interesse an Selbstachtung' sprechen. 
3. Erweiterte Reziprozität: Seit Marcel Mauss (1968) die sozialen Funktionen des Austausches von Geschenken analysiert hat, lassen sich eine Vielzahl anscheinend altruistischer Verhaltensweisen als Systeme einer erweiterten Reziprozität interpretieren, die weder dem Typus marktmäßigen Tausches noch demjenigen herrschaftlicher Kontrolle entsprechen. Sie sind vor allem in traditionalen Gesellschaften vielfach nachgewiesen, können aber auch in modernen Gesellschaften eine nicht unerhebliche Rolle spielen, beispielsweise bei der Begründung wohlfahrtsstaatlicher Umverteilungssysteme. ${ }^{77}$ Charakteristisch für diesen Typus ist eine tauschtheoretische Begründung solidarischen Verhaltens: Der Verzicht auf eigennützige Handlungsweisen wird mit einer indirekten oder vermittelten wechselseitigen Abhängigkeit begründet.

4. Kollektivitätsorientiertes Verhalten: Die klassische Formel der Solidarität: „Einer für Alle - Alle für Einen" gewinnt soziale Plausibilität nur unter der Voraussetzung klarer Gruppengrenzen und wirksamer sozialer Kontrolle unter den Gruppenmitgliedern, wie wir sie unter traditionalen Bedingungen im Regelfalle unterstellen können. Natürlich handelt es sich bei dieser ,starken' Solidaritätsformel, deren Ursprung auf das römische Haftungsrecht und damit auf einen sehr spezifischen Tatbestand zurückgeht, in ihrer verallgemeinerten Form stets um eine Idealisierung. Die im 19. Jahrhundert aufkommende ,Gemeinschafts'-Rhetorik, welche heute bei den weniger reflektierten Kommunitaristen Urständ feiert, verklärte die Vergangenheit. Soweit es sich nicht um rechtlich erzwingbare Solidarhaftung handelt, sind radikale Formen, gemeinschaftlichen " Handelns sowohl für die Vergangenheit wie auch für die Gegenwart nur in hoch emotionalisierten Kontexten zu erwarten. Infolge der gesellschaftlichen Differenzierungsprozessen und der damit verbundenen Vervielfachung von Gruppenzugehörigkeiten sowie der daraus resultierenden Tendenz zur Individualisierung sind Situationen einer umfassenden ,gemeinschaftlichen ' Gruppenbindung heute selten geworden. Die meisten Menschen leben „in sich kreuzenden sozialen Kreisen“ (G. Simmel) und damit in vielfältigen Gruppenbeziehungen. Zur Vermeidung irreführender Idealisierungen wird dieser Typus von Solidarität hier nicht als ,gemeinschaftsorientiert ${ }^{*}$ sondern als ,kollektivitätsorientiert' bezeichnet. ${ }^{78}$ Kollektivitätsorientiertes Handeln läßt sich auch heute in nahezu allen dauerhaften sozialen Verbindungen beobachten, in denen interpersonelle Abhängigkeiten und soziale Anerkennung eine bedeutende Rolle spielen. Es beruht auf der Erwartung, daß die Mitglieder eines Kollektivs konsequent ein mehr oder weniger explizites Kollektivinteresse bei ihren Entscheidungen und Handlungen berücksichtigen, das über ihre Rechtspflichten hinausgeht. Von bloßer Loyalität unterscheidet sich dieser Typus durch ein aktives Eintreten für ,gemeinsame Interessen', vom altruistischen Verhalten durch eine zum mindesten partielle Überlappung oder ein „,Verschwimmen" (Wiesenthal) von Eigen- und Kollektivinteresse. Die Abgrenzung zum Typus, erweiterte Reziprozität' ergibt sich aus der deutlicheren kollektiven Definition

77 Die Kategorie der erweiterten Reziprozität wurde vor allem von Karl Polanyi ausgearbeitet; vgl zusammenfassend Humphreys 1979, S. 46 ff.

${ }^{78}$ Der Typus deckt sich jedoch weitgehend mit dem, was in der Literatur als ,gemeinschaftliche* Form von Koordination behandelt wird; vgl. zusammenfassend Wiesenthal 2000. 
von gemeinsamen Interessen und damit einer direkteren, unmittelbareren Verknüpfung der Handlungsfolgen. ${ }^{79}$

Der Sinn dieser vierfachen und auf dem gewählten Niveau der Abstraktion m.E. vollständigen Typologie wird deutlicher, wenn wir berücksichtigen, daß die Klassifikation auch eine Rekonstruktion gesellschaftlich verbreiteter Solidaritätsbegründungen darstellt. Wer immer Menschen von eigennützigem Handeln abhalten will, hat sich - so die These - eines dieser vier Begründungsmuster - Loyalität, Altruismus, Reziprozität oder Gemeinsamkeit - zu bedienen. Quintum non datur. Die Zuordnung der Empirie bleibt wie immer in solchen Fällen mit Unschärfen behaftet, aber die Typologie selbst ist trennscharf.

\subsubsection{Solidarität als Form sozialer Steuerung}

Der hier vorgestellte Solidaritätsbegriff orientiert sich antithetisch am Begriff egoistischer Interessenverfolgung, in ähnlicher Weise wie der Begriff des, Sozialen' im 19. Jahrhundert. ${ }^{80}$ Er hat jedoch einen präziseren und damit eingeschränkteren Bedeutungsumfang. Er stellt insbesondere in Rechnung, daß in modernen Gesellschaften individuelles Handeln in der Regel nicht mehr unmittelbar, gesellschaftsrelevant ${ }^{\text {‘ }}$ wird, sondern nur vermittelt über die Mitgliedschaft in Organisationen und über Transaktionen in Inter-Organisationsbeziehungen, welche ihrerseits ausschließlich formalen Regeln folgen. Organisationen kennen keine Gefühle und sind damit auch für „,moral sentiments“ (A. Smith) nicht zugänglich. ,Solidarität " als Steuerungsmodus operiert demzufolge essentiell auf der personalen und interaktiven, nicht der organisatorischen Ebene.

Aber das kollektive Handeln von Organisationen ist selbstverständlich auch von zwischenmenschlichen Interaktionen und individuellen Entscheidungen abhängig. Deren

79 Die Literatur zu ,neuen Solidaritätsformen' (z.B. Hondrich u. Koch-Arzberger 1992; Frankenberg 1997) betont allzu sehr den zeitlich begrenzten und punktuellen Charakter moderner Solidaritäten. Diese Beobachtung orientiert sich vor allem am Wandel der Engagementbereitschaft jüngerer Menschen, die nur noch schwer für ein längerfristiges ,Ehrenamt ' oder gar für lebenslange Bindungen (z.B. in Orden), aber durchaus für projektförmige und damit zielorientierte und zugleich befristete Engagements zu begeistern sind. In ähnlicher Weise werden die herkömmlichen Vereine den ,sozialen Bewegungen' gegenübergestellt. Zum einen wird hier etwas Richtiges gesehen, nämlich die Erosion traditional begründeter und i.d.R. mit hoher Seßhaftigkeit verbundener Formen von Solidarität und der gesteigerte Individualisierungsgrad moderner Sozialverhältnisse. ,Soziale Bewegungen" sind gemäß obiger Klassifikation ein Kontext sowohl für altruistisches als auch für kollektivitätsorientiertes Handeln, je nach den dominierenden Sinnelementen advokatorischer oder eigener Interessenvertretung. Charakteristische Kontexte des kollektivitätsorientierten Handelns sind heute eher in Subeinheiten von Organisationen zu sehen, in denen sich kollektive Interessen auch in Konkurrenz zu anderen Subeinheiten herausbilden. Überhaupt dürfte Gruppenkonkurrenz heute ein typischer Kontext für die Entstehung kollektivitätsorientierten Verhaltens sein. Die Ambivalenz solidaritätsorientierter Diskurse (vgl. z.B. Bayertz 1998, S. 48 ff; Zoll 2000) resultiert aus dem Umstand, daß Solidarität weit stabiler in überschaubaren Gruppen mit partikularistischen Interessen entsteht als in den mit besonderen Hoffnungen verbundenen advokatorischen Assoziationen und sozialen Bewegungen.

${ }^{80} \mathrm{Vgl}$. Abschnitt 1.2 . 
Bandbreite wird durch die Prämissen der Organisation eingeschränkt, aber im Ergebnis nicht bestimmt.

„Individuen agieren im betrieblichen Kontext einerseits als Positionsinhaber und Rollenträger auf der Basis formalisierter und damit stets selektiver Verhaltenserwartungen. Andererseits bringen sie jedoch trotz der geforderten Trennung von Organisationszwecken und persönlichen Aspirationen immer wieder Ideen, Gefühle, Motive und Verhaltensweisen ein, die außerbetrieblich entstanden und geprägt sind. “ 81

Insofern können moralische Intentionen auch durch Organisationen zur Geltung gebracht werden, doch bedürfen sie für ihre Wirksamkeit i.d.R. einer stets erneuerten Zufuhr moralischer Motive von Seiten ihrer Mitglieder. Eine gewisse Stabilisierung moralischer Intentionen auf der Organisationsebene kann darüber hinaus durch deren Fixierung in der Organisationsprogrammatik gelingen, sei es in der Form wertrationaler Organisationszwecke, sei es in der Form einer ethisierten Unternehmenskultur. Vor allem aber reagieren Organisationen sensibel auf Veränderungen in ihrer Umwelt: „Menschenrechtssensibilität verändert das Investitionsklima, und dann reagieren sogar Vorstandsetagen. " 82

Der Bezug auf emotionalisierbare, ,sympathetische ${ }^{\text {Personen }}{ }^{83}$ ist natürlich auch für traditionale Formen der Solidarität charakteristisch, war aber dort selbstverständlich, da alle relevatusy Sozialbeziehungen interpersonellen Charakter trugen und sich innerhalb einer gemeinsamen Lebenswelt vollzogen. Das Neue ist die organisatorische Fragmentierung der lebensweltlichen Wirklichkeit und damit die Pluralisierung der individuellen Lebenswelten, so daß alle sozialen Beziehungen zwangsläufig ihren umfassenden Charakter verlieren und selektiv werden. Nicht nur hierarchische und marktmäßige, sondern auch Solidarität begünstigende Kontexte verlieren dadurch an umfassender, diffuser ,Gemeinschaftlichkeit ${ }^{6}{ }^{84}$ Solidarisches Handeln entsteht heute häufig nicht aufgrund dauerhaft feststehender, gemeinsamer Ziele, sondern ,in Akten des Verhandelns und Ausbalancierens unterschiedlicher Normen und Interessen". ${ }^{85}$

Worin aber besteht der spezifische Charakter von Solidarität als Steuerungsform menschlichen Handelns? Zur Beantwortung dieser Frage muß zunächst das steuerungstheoretische Bezugsproblem geklärt werden. In diesem Zusammenhang konkurrieren unterschiedliche Ansätze, wobei vor allem zwischen einem direktiven und einem Formen der Selbststeuerung mit umfassenden Begriff von Steuerung zu unterscheiden ist. ${ }^{86}$ Hier wird ein breiter Steuerungsbegriff zugrunde gelegt, welcher alle im Zusammenspiel von Institutionalisierung und Interaktion typisierbaren Formen der Handlungskoordination als ,Steuerung' begreift. Dabei werden fünf Typen der Koordination von Handlungen unterschieden, nämlich Hierarchie, Markt, Solida-

81 Hegner 1997, S. 310.

${ }^{82}$ Brunkhorst 2000, S. 11.

83 Zur Sympathie als Grundlage moralischer Gefühle vgl. Smith 1977, S. 1 ff; zur Interpretation im Kontext einer Analyse moderner Solidaritätsformen vgl. Kaufmann 1984, S. 173-179.

84 Diesen Gesichtspunkt vernachlässigt der im übrigen zu unseren Überlegungen komplementäre Aufsatz von Wiesenthal 2000 .

${ }^{85}$ Gabriel/Herlth/Strohmeier 1997, S. 25.

${ }^{86}$ Für Überblicke vgl. Gotsch 1987 und Ulrich 1994, insb. S. 84-92. 
rität, Professionalität und Korporatismus. Ihnen allen ist gemeinsam, daß sie prinzipiell die drei Funktionsbedingungen dauerhafter Handlungskoordination gewährleisten, nämlich die Normierung von Standards adäquater Leistungserbringung (Guidance), die Sicherung von Konformität der beteiligten (kollektiven oder individuellen) Akteure (Control) und die bewertende Kommunikation über Ergebnisse von Handlungsverkettungen (Evaluation). Dabei unterscheiden sich die fünf Typen hinsichtlich der Art und Weise der durch sie bewirkten Handlungskoordination sowie hinsichtlich der dominanten Motive konformen Verhaltens, wie auch hinsichtlich der Kommunikationsform. $^{87}$

Im vorliegenden Zusammenhang interessiert allein der Steuerungstypus der Solidarität. ${ }^{88}$ Solidarität erscheint vor dem Hintergrund moderner Diagnosen einer rationalisierten, individualisierten und tendenziell anonymen Gesellschaft ein zunächst höchst unwahrscheinliches bzw. zum Aussterben verurteiltes Phänomen, und es sind derzeit vor allem dem amerikanischen Kommunitarismus nahestehende Autoren, die einen Rückgang des „sozialen Kapitals“ in der Gesellschaft der Vereinigten Staaten diagnostizieren. ${ }^{89}$ Hier wird dagegen die These vertreten, daß gerade unter hoch individualisierten gesellschaftlichen Lebensbedingungen individuelle Bedürfnisse nach Anerkennung und identitätsförderliche Selbstwertgefühle eine neuartige motivationale Voraussetzung solidarischen Handelns bilden, analog der Furcht vor Nachteilen in hierarchischen Beziehungen oder dem ökonomischen Interesse in Marktbeziehungen. Die soziale Voraussetzung für das Wirksamwerden dieser motivationalen Disposition ist typischerweise eine interpersonelle Vernetzung im Rahmen normativer, Wahlverwandtschaften" oder ,Gesinnungsgemeinschaften ${ }^{6}{ }^{90}$ In der Weberschen Typologie sozialen Handelns sind typisch moderne Formen von Solidarität dem wertrationalen, nicht dem traditionellen oder emotionalen Typus zuzuordnen, obwohl natürlich auch derartige Solidaritätsformen in bestimmten Kontexten persistieren oder neu entstehen können. Wir haben bereits auf die Wirksamkeit von Solidarität im Rahmen überschaubarer Organisationen (z.B. Vereine) oder Organisationseinheiten hingewiesen, im Rahmen derer - wenngleich häufig prekäre - Formen der Traditionsbildung zu beobachten sind. ${ }^{91}$

${ }^{87}$ Vgl. Kaufmann 1991a, insb. die Übersicht S. 228

88 Helmut Willke 1995, S. 91, wendet sich gegen die Einbeziehung von Solidarität in eine Steuerungstheorie moderner Gesellschaften. Für ihn ist Solidarität die nur ,in verbliebenen Winkeln der Lebenswelt noch [vorhandene, F.-X.K.] herzerfrischende Irrationalität altruistischer, sorgender, beschützender und bisweilen aufopfernder Handlungsmuster, die einer rationalistischen oder ökonomistischen Sichtweise unbegreiflich bleiben müssen." Unsere Ausführungen sollen demgegenüber zeigen, daß, Solidarität' gerade nicht nur „ein ,Überleben' vormoderner, gemeinschaftlicher Koordinationsformen [...] zur Abstützung und Unterfütterung der anonymen, modernen Formen" (ebd.) ist, sondern in neuen Kontexten und mit veränderten Motivationen auch als typisch moderne Koordinationsform interpretiert werden kann.

89 Prominent Putnam 1995

90 Gabriel/Herlth/Strohmeier 1997, S. 20 ff.

91 Vgl. Fn. 23; dieser Typus steht im Zentrum des „Koordinationsmechanismus Gemeinschaft“ bei Wiesenthal 2000, womit ein deutlich engerer Phänomenbereich als der hier anvisierte abgedeckt wird. 
Primär emotionale Vergemeinschaftungsformen persistieren vor allem im Bereich von Familie und Verwandtschaft. ${ }^{92}$

Solidarische Handlungskoordination unterscheidet sich von allen anderen Typen der Handlungskoordination durch ihren informellen, nur an implizite Regeln gebundenen Charakter. Wir können dabei phänomenologisch zwischen alltäglichen und emphatischen Erscheinungsformen unterscheiden. Vor allem in der Form von Loyalität, aber auch in der Anerkennung von Reziprozitätsverhältnissen operiert Solidarität alltäglich und implizit, sie ist „ein leistungssteigerndes Additiv für jeglichen Modus sozialer Interaktion". ${ }^{93}$ Sie ist orientiert an etablierten Standards adäquater Leistungserbringung und evaluiert das Verhalten der Interaktionspartner nach impliziten Standards von,,Treu und Glauben" oder der "Schicklichkeit". ${ }^{94}$ Wer Solidaritätserwartungen dieser Art verletzt, riskiert seinen ,Ruf", Sanktionen erfolgen meist indirekt. Positive Anerkennung ist hier die Ausnahme.

Emphatische oder explizite Formen der Solidarität beruhen entweder auf wertrationalen Handlungsorientierungen oder auf kollektiver Efferveszenz, wie sie insbesondere in Krisensituationen oder bei Katastrophen auftritt. Der zweite Fall kann sowohl unter Bedingungen traditionaler Vergemeinschaftung als auch unter den hoch individualisierten Bedingungen der Moderne auftreten, auch Massenbewegungen sind hier einzuordnen. Typisch modern erscheinen dagegen freie Assoziationen mit gemeinsamen Zielsetzungen, von den bürgerlichen Geselligkeitsvereinen über Selbsthilfegruppen bis zu sozialen Bewegungen mit emphatisch wertrationalen Zielen. Je geringer der Organisationsgrad, desto deutlicher tritt in der Regel das emphatische Moment normativer Gemeinsamkeiten in den Vordergrund. ${ }^{95}$ Dabei werden Standards adäquater Leistungserbringung häufig in deliberativen Prozessen explizit formuliert und ihre Erreichung evaluiert. Derartige Prozesse fundieren zugleich nicht selten die Reputation der Beteiligten.

Die Beschwörung bestimmter, als gemeinsam postulierter Werte, welche i.d.R. auch kulturell verankert sind, ist heute konstitutiv für öffentliche Solidaritätsdiskurse, und diese zeichnen sich vielfach durch hohe Reichweite aus, sei es in zeitlicher (,Nachhaltigkeit', ,Zukünftige Generationen'), sei es in sozialer Hinsicht (Einbezug des ,Andersartigen' bzw. ,Fremden $\left.{ }^{6}\right){ }^{96}$ in sachlicher Hinsicht bleiben sie jedoch meist recht unbestimmt. ${ }^{97}$ Genau genommen wird oft ,Solidarität ${ }^{\circ}$ selbst als Eigenwert postuliert und - wie schon der Vorgängerbegriff ,Brüderlichkeit ${ }^{\star}$ - auf einer Stufe mit ,Freiheit" oder ,Gleichheit ${ }^{\star}$ verhandelt. Unsere Überlegungen sollen dazu dienen, ,Solidarität' ${ }^{\varsigma}$ aus den lichten Höhen des bloß Wünschbaren auf den Boden sozialer Tatsachen herunterzuholen. Wir verstehen Solidarität als Typus sozialen Handelns, dessen Auftreten von

92 Zu Formen, Problemen und Wirkungen familialer Solidarität im Kontext moderner Vergesellschaftung vgl. von Schweitzer 1997; Lüscher 1997; Huinink 1997 und Schulze/Künzler 1997.

93 Wiesenthal 2000, S. 48.

94 Adam Smith spricht von „sense of propriety“, was in der deutschen Ausgabe bald mit „Schicklichkeit", bald mit „Gefühl für das sittlich Richtige“ übersetzt wird.

${ }^{95}$ Zum Zusammenhang von Moralisierung und Mobilisierung bei sozialen Bewegungen vgl. Hellmann 1996, S. 231 ff. Zur dimensionalen Analyse von Selbsthilfegruppen vgl. Halves/Wetendorf 1986.

${ }^{96}$ Hiezu grundsätzlich Brunkhorst 1997.

97 Vgl. Zoll 2000, S. $144 \mathrm{ff}$. 
bestimmten, soziologisch aufzuklärenden Bedingungen abhängig ist. Auf der Individualebene erscheint die Solidaritätsbereitschaft von einer ,moralischen" Gefühlslage abhängig, d.h. von gefühlter Pflicht oder engagierter Neigung, bis hin zur Begeisterung. Derartige Gefühlslagen stehen in der Regel in einem kontingenten Verhältnis zu den individuellen Interessenlagen, sie brauchen diesen keineswegs zu widersprechen; aber der immer wieder thematisierte, weil allein problematische Fall ist der Konflikt zwischen wahrgenommenem Eigeninteresse einerseits und Pflicht oder Neigung andererseits. Für die theoretische Analyse kann jedoch nicht der Konfliktfall, sondern nur die Eigenart der Handlungsmotivation selbst von Bedeutung sein.

Individuen unterscheiden sich schon von Temperament und Sozialisation her hinsichtlich ihrer Disposition, Pflichten anzuerkennen oder Engagements zu übernehmen, doch die Variation generalisierter subjektiver Einstellungen steht hier nicht zur Debatte. ${ }^{98}$ In steuerungstheoretischer Hinsicht stellt sich die Frage nach den institutionellen oder kontextuellen Bedingungen, welche geeignet sind, intrinsische Pflichterfüllung oder Engagement zu aktualisieren. Sie müssen grundsätzlich dreierlei Eigenschaften aufweisen, nämlich zum einen die Normativität und zum Anderen die Aktualität der Forderung nach solidarischem Handeln den Akteuren plausibel machen, und schließlich positive oder negative Anreize zu einem entsprechenden Verhalten implizieren. Diese Anreize sind definitionsgemäß weder vom Typus machtbewehrter Drohung (,Hierarchie") noch vom Typus ökonomischer Vor- und Nachteile (,Markt'). Typischerweise handelt es sich um immaterielle Anreize, welche das Bedürfnis nach Anerkennung befriedigen oder verletzen: Die Kommunikation von Achtung oder Mißachtung ist typisch für ,moralische ${ }^{\text {Kommunikation. }}{ }^{99}$ Darüber hinaus ist jedoch insbesondere für moderne Formen der Solidaritätsbereitschaft noch eine intrinsische Disposition zur Bestätigung von Selbstachtung mittels wertrationalen Handelns zu postulieren, welche jedoch keineswegs monadisch, sondern ihrerseits als durch sozial gestützte Prozesse der Identitätsbildung und -bestätigung vermittelt zu verstehen ist. Neben der Entstehung von Wertbindungen im Rahmen von Sozialisationsprozessen ist hier unter hoch individualisierten Bedingungen menschlichen Zusammenlebens insbesondere auch an freiwillige Zusammenschlüsse im Rahmen von ,Wahlverwandtschaften“ zu denken.

Herkömmliche, gemeinschaftliche' Formen der Solidarität beruhen auf dichten, in funktionaler Hinsicht diffusen sozialen Beziehungen. Aus Beobachtersicht nahezu beliebige Ereignisse können Solidarisierungen, aber auch Konflikte auslösen. Moderne Formen der Solidarität sind dagegen in hohem Maße kontextabhängig, und zwar in doppelter Hinsicht: Zum einen vollzieht sich soziales Handeln regelmäßig in institutionell und/oder organisatorisch definierten Kontexten, aus denen sich der Horizont jeglicher Regel- oder Kollektivitätsorientierung ergibt. Zum anderen erfordern diese ,settings` nur im Ausnahmefall explizit solidarisches Handeln, da ihre Stabilität natürlich erfordert, daß im alltäglichen Verkehr auch Eigeninteressen mitbefriedigt werden. Es bedarf deshalb nicht nur gemeinsamer Wertorientierungen, sondern auch geteilter

\footnotetext{
98 Man könnte allerdings pädagogische, bzw. familien- oder bildungspolitische Überlegungen zur allgemeinen Förderung von Pflichtbewußtsein oder/und Engagementbereitschaft anstellen, was jedoch den vorliegenden steuerungstheoretischen Diskurs sprengen würde.

99 Vgl. Luhmann 1978.
} 
außeralltäglicher Situationsdefinitionen, um emphatische Solidarität zu mobilisieren. So aktualisiert sich Solidarität als Kollektivitätsorientierung vor allem entweder in Krisensituationen oder in Situationen enthusiastischer Hoffnungen.

\section{Zusammenfassung}

Als ,Sozialpolitik' wurde die Suche nach programmatischen und institutionellen Antworten auf die ,sozialen' Probleme bezeichnet, welche durch den Verlust der zwar ärmlichen, aber doch einigermaßen verbindlichen Schutzrechte des Zunft- und Feudalsystems und die von der Liberalisierung der Wirtschaft ausgelöste Mobilität hervorgebracht worden waren (Abschnitt 1.2). Die Industrialisierung hat diese Problemlagen mitgeprägt, doch brachte sie gleichzeitig auch die technischen und wirtschaftlichen Fortschritte, welche die Überwindung von Armut, die Verbesserung der städtischen und betrieblichen Hygiene sowie eine die Lebenschancen auf breiter Front verbessernde ,soziale Verwaltung' (von Stein) erst ermöglichten. Diese Transformationsprozesse haben gleichzeitig Anlaß zu einer tiefgreifenden Veränderung der gesellschaftstheoretischen Auffassungen gegeben (Abschnitt 1.1).

Unter Stichworten wie ,Risikogesellschaft', ,Individualisierung' oder ,Globalisierung werden heute neue Problemlagen thematisiert, welche den Rahmen wohlfahrtsstaatlicher Politik sprengen. Da Lösungen weder durch Marktkräfte - die häufig geradezu als Krisenerzeuger gelten - noch durch die etablierten Formen von Politik und Verwaltung in Sicht sind, richtet sich die Hoffnung vielfach auf die Mobilisierung sozialmoralischer Ressourcen, um der anstehenden Probleme Herr zu werden. Die erneute Thematisierung des Zusammenhangs von ,Gemeinwohl' und, Gemeinsinn' gibt dem Ausdruck.

Sozialmoralische Ressourcen haben in der Entstehungs- und Formulierungsphase von Sozialpolitik eine erhebliche Rolle gespielt, und zwar sowohl in der Praxis - von der Wohltätigkeit über die Solidarität der Arbeiterbewegung bis zum Engagement von Politikern und Verwaltungsleuten -, als auch in verwissenschaftlichen Diskursen (Abschnitt 1.3) Allerdings hat sich die Verknüpfung von moralischen Forderungen und staatlicher Politik als wenig stabil erwiesen. Die Sozialpolitik verlor den „Kontakt mit der Seele des einfachen Mannes“ (G. Briefs), und die Hoffnungen richteten sich nun zunehmend auf Verhandlungslösungen zwischen Gewerkschaften und Arbeitgeberverbänden, welche einen allgemeinen moralisch-politischen Konsens überflüssig machen sollten (Abschnitt 1.4). Damit verschob sich die Problematik der Konsensfindung auf die partikularistische Ebene der Tarifparteien und deren interne Bindungsfähigkeit. Im Zuge der Tertiarisierung der Wirtschaft und der Individualisierung der Lebensbezüge sinkt auch diese Bindungsfähigkeit, und parallel dazu verschob sich das sozialpolitische Paradigma vom Klassenkonflikt zur Verteilungsproblematik. Damit geriet die Perspektive von Gemeinsinn und Gemeinwohl ganz aus dem Fokus der Sozialpolitik (Abschnitt 1.5).

Im zweiten Teil wurde diese Perspektive selbst in Frage gestellt. Zwar ist es plausibel, daß auch moderne Gesellschaften weder auf die Gewährleistung von Bedingungen des Gemeinwohls noch auf die Wirksamkeit sozialmoralischer Ressourcen ver- 
zichten können, aber der in der traditionellen politischen Theorie postulierte direkte Zusammenhang zwischen beiden Größen hat sich im Zuge der Modernisierung aufgelöst. Die Vervielfältigung der Solidaritätshorizonte im Zuge der Steigerung gesellschaftlicher Komplexität sowohl in funktionaler als auch in politischer Hinsicht zwingt zu einer Reformulierung der Problematik (Abschnitt 2.1). Wir halten dabei am Begriff des Gemeinwohls fest, schränken ihn jedoch auf die allgemein zustimmungsfähigen Normen moderner Verfassungen ein (Abschnitt 2.2). Im Horizont des insoweit durch die Anerkennung von Menschenrechten inhaltlich und durch die Gewährleistung von Volkssouveränität und Rechtsstaatlichkeit prozedural gewährleisteten Gemeinwohls bleiben politische Entscheidungen eine Frage partikulärer Prozesse der Solidarisierung und mehr oder weniger konfliktiver Aushandlung, wobei sich die Konflikte durch keinerlei übergreifenden, Gemeinsinn' überwinden lassen. Die Mobilisierung sozialmoralischer Ressourcen erfolgt nunmehr notwendigerweise auf partikulären Ebenen, so daß die Bezeichnung als ,Gemeinsinn" mißverständlich wäre. Ebenso mißverständlich ist der Begriff der ,Gemeinschaft' zur Kennzeichnung der institutionellen und kontextuellen Form einer Mobilisierung sozialmoralischer Ressourcen (Abschnitt 2.3.1). Wir haben ihn deshalb durch den ebenfalls traditionsreichen, jedoch im Hinblick auf die Vervielfältigung von Handlungskontexten aussagekräftigeren Begriff der ,Solidarität" ersetzt und vier verschiedene Diskurse der Solidaritätsbegründung - Loyalität, erweiterte Reziprozität, Altruismus und Kollektivitätsorientierung - unterschieden (Abschnitt 2.3.2). Solidarität wird schließlich als spezifische Koordinationsform (Steuerungstypus) sozialen Handelns expliziert, die auf der Ebene interpersoneller Beziehungen ihre spezifische Wirksamkeit entfaltet und sich gerade darin von allen anderen Koordinationsformen unterscheidet. Solidarität erweist sich daher als ein wichtiges Bindeglied zwischen der individuellen und der formal organisierten und in der Regel rechtlich verfaßten Ebene sozialen Handelns in modernen Gesellschaften (Abschnitt 2.3.3).

Es ist nicht $\mathrm{zu}$ bestreiten, daß die verrechtlichten und hoch organisierten Formen politisch-administrativer, aber auch privat-unternehmerischer Problembearbeitung für die Bearbeitung zahlreicher aktueller Problemlagen nur teilweise geeignet sind, und das gilt insbesondere für den Bereich der personenbezogenen Dienstleistungen. Es kommt daher nicht von ungefähr, daß von ,Solidarität' vor allem hinsichtlich des sog. ,Dritten Sektors ${ }^{6}$ Großes erwartet wird. ${ }^{100}$ Aber auch im Bereich von staatsübergreifenden, tendenziell ,weltweiten' Problemen wie Frieden, Umwelt, ,Dritte Welt' oder riskanten Technologien, haben sich Aktionsgruppen und soziale Bewegungen gebildet, die zugleich wertrational argumentieren und allgemeine Gefährdungen als Situationsdefinition suggerieren, um auf diese Weise Solidarität nicht nur unter den Aktivisten, sondern darüber hinaus in möglichst weiten Kreisen der Bevölkerung zu erzeugen. Auch wenn nicht damit zu rechnen ist, daß derartige Mobilisierungen sozialmoralischer Ressourcen ihre erklärten Ziele gegen den Widerstand von Interessengruppen und die kompartimentierte Borniertheit der institutionellen Politik unmittelbar werden erreichen können, so ist ihr Einfluß auf die öffentliche Meinung und einen allmählichen kollektiven Bewußtseinswandel doch nicht zu unterschätzen. Ob und inwieweit derartige Solidarisierungen und

${ }^{100}$ Vgl. z.B. Frankenberg 1997, S. 206 ff. 
Mobilisierungen dem wie auch immer definierten Gemeinwohl dienen, wird jedoch stets eine politisch und moralisch umstrittene Frage bleiben.

\section{Literaturverzeichnis}

Achinger, H. (1939), Sozialpolitik und Fürsorge. Ein Abgrenzungsversuch, begründet aus den Ursachen der Notstände, Berlin.

Backhaus, J. G. (1993), Gustav von Schmoller und die Probleme von heute, Berlin.

Bayertz, K. (1998), Begriff und Problem der Solidarität, in: Solidarität - Begriff und Problem, hg. v. K. Bayertz, Frankfurt/M., S. 11-53.

Berger, P. L./Luckmann, T. (1969), Die gesellschaftliche Konstruktion der Wirklichkeit. Eine Theorie der Wissenssoziologie, Frankfurt/M.

Berger, P. L. (Hg., 1997), Die Grenzen der Gemeinschaft. Konflikt und Vermittlung in pluralistischen Gesellschaften, Gütersloh.

Blum, R. (1969), Soziale Marktwirtschaft - Wirtschaftspolitik zwischen Neoliberalismus und Ordoliberaiismus, Tübingen.

Bode, I. (1999), Solidarität im Vorsorgestaat. Der französische Weg sozialer Sicherung und Gesundheitsversorgung, Frankfurt/M./New York.

Bourgeois, L. (1896), Solidarité, Paris.

Bortkiewicz, L. v. (1899), Der Begriff ,Sozialpolitik", in: Jahrbücher für Nationalökonomie und Statistik, 72, S. 332-349.

Briefs, G. (1923), Zur Krisis in der Sozialpolitik, in: Kölner sozialpolitische Vierteljahresschrift 3, S. $1-16$.

Brumlik, M./Brunkhorst, H. (Hg., 1993), Gemeinschaft und Gerechtigkeit, Frankfurt/M.

Brunkhorst, H. (1997), Solidarität unter Fremden, Frankfurt/M.

Brunkhorst, H. (2000), Globale Solidarität. Inklusionsprobleme der modernen Gesellschaft, Ms.

Donzelot, J. (1984), L'invention du social. Essai sur le déclin des passions politiques, Paris.

Frankenberg, G. (1997), Die Verfassung der Republik. Autorität und Solidarität in der Zivilgesellschaft, Frankfurt/M.

Gabriel, K. u. a. (Hg., 1997), Modernität und Solidarität. Konsequenzen gesellschaftlicher Modernisierung, Freiburg i. Br.

Gabriel, K/Herlth, A./Strohmeier, K. P. (1997), Solidarität unter den Bedingungen entfalteter Modernität, in: Modernität und Solidarität. Konsequenzen gesellschaftlicher Modernisierung, hg. v. K. Gabriel u. a., Freiburg i. Br., S. 13-27.

Geser, H. (1990), Organisationen als soziale Akteure, in: Zeitschrift für Soziologie 19, S. 401-417.

Gotsch, W. (1987), ,Soziale Steuerung ${ }^{c}$ - zum fehlenden Konzept einer Debatte, in: Dezentrale Gesellschaftssteuerung. Probleme der Integration polyzentrischer Gesellschaft, hg. v. M. Glagow u. H. Willke, Pfaffenweiler, S. 27-44.

Gretschmann, K. (1991), Solidarity and Markets Reconsidered: Cum, Versus, or What?, in: The Public Sector. Challenge for Coordination and Learning, hg. v. F.-X. Kaufmann, Berlin/New York, S. $395-415$

Grün, K. (1974), Die soziale Bewegung in Frankreich und Belgien [1845], Hildesheim.

Gurvitch, G. (1972), L'idée du droit social [1932], Aalen.

Halves, E./Wetendorf, H.-W. (1986), ,Natürlich hat sich die Gruppe mit der Zeit verändert ..." Verläufe von Selbsthilfegruppen, in: Wissen ist Macht - Eigenständig durch Selbsthilfe in Gruppen, hg. v. A. Trojan, Frankfurt/M., S. 137-162.

Hatzfeld, H. (1971), Du paupérisme à la sécurité sociale, 1850-1940, Paris. 
Hayward, J. E. S. (1961), The Official Social Philosophy of the French Third Republic: Léon Bourgeois and Solidarism, in: International Review of Social History 6, S. 19-48.

Hegel, Georg Wilhelm Friedrich $\left(1955^{4}\right)$, Grundlinien der Philosophie des Rechts [1821], hg. v. Johannes Hoffmeister, Hamburg.

Hegner, F. (1985/1991), Comparing Solidarity, Hierarchy, and Markets: Institutional Arrangements for the Coordination of Actions, in: The Public Sector. Challenge for Coordination and Learning, hg. v. F.-X. Kaufmann, Berlin/New York, S. 417-439.

Hegner, F. (1997), Besser kluger Egoismus als scheinbarer Altruismus. Loyalität, Vertrauen und Reziprozität als Koordinationsmechanismen, in: Modernität und Solidarität. Konsequenzen gesellschaftlicher Modernisierung, hg. v. K. Gabriel u. a., Freiburg i. Br., S. 309-325.

Heimann, E. (1980), Soziale Theorie des Kapitalismus - Theorie der Sozialpolitik [1929], Frankfurt/M.

Hellmann, K.-U. (1996), Systemtheorie und neue soziale Bewegungen. Identitätsprobleme in der Risikogesellschaft, Opladen.

Hirschman, A. O. (1970), Exit, voice, and loyalty: responses to decline in firms, organizations, and states, Cambridge Mass.

Höffe, O. (1991), Eine entmoralisierte Moral: Zur Ethik der modernen Politik, in: Politische Vierteijahresschrift 32, S. 302-316.

Hondrich, K.-O./Koch-Arzberger, C. (1992), Solidarität in der modernen Gesellschaft, Frankfurt/M.

Honneth, A. (Hg., 1993), Kommunitarismus: eine Debatte über die moralischen Grundlagen moderner Gesellschaften, Frankfurt/M./New York.

Huinink, J. (1997), Elternschaft in der modernen Gesellschaft, in: Modernität und Solidarität. Konsequenzen gesellschaftlicher Modernisierung, hg. v. K. Gabriel u. a., Freiburg i. Br., S. 79-90.

Humphreys, S. C. (1979), Einleitung: Geschichte, Volkswirtschaft und Anthropologie: Das Werk Karl Polanyis, in: Ökonomie und Gesellschaft, Karl Polanyi, Frankfurt/M., S. 7-59.

Kaufmann, F.-X. (1982), Elemente einer soziologischen Theorie sozialpolitischer Intervention, in: Staatliche Sozialpolitik und Familie, hg. v. F.-X. Kaufmann, München/Wien, S. 49-86.

Kaufmann, F.-X. (1984), Solidarität als Steuerungsform - Erklärungsansätze bei Adam Smith, in: Markt, Staat und Solidarität bei Adam Smith, hg. v. F.-X. Kaufmann u. H.-G. Krüsselberg, Frankfurt/M./New York, S. 158-184.

Kaufmann, F.-X. (1988), Christentum und Wohlfahrtsstaat, in: Zeitschrift für Sozialreform 34, S. 65-89.

Kaufmann, F.-X. (Hg., 1991), The Public Sector. Challenge for Coordination and Learning, Berlin/New York.

Kaufmann, F,-X. (1991a), The Relationship between Guidance, Control, and Evaluation, in: The Public Sector. Challenge for Coordination and Learning, hg. v. F.-X. Kaufmann, Berlin/New York, S. 213-234.

Kaufmann, F.-X. (1992), Der Ruf nach Verantwortung. Risiko und Ethik in einer unüberschaubaren Welt, Freiburg i. Br.

Kaufmann, F.-X. (1998), Globalisierung und Gesellschaft, in: Aus Politik und Zeitgeschichte, Bd. 18, S. 3-10.

Kaufmann, F.-X. (2001), Der Begriff Sozialpolitik und seine wissenschaftliche Deutung, in: Geschichte der Sozialpolitik in Deutschland seit 1945, hg. v. Bundesministerium für Arbeit und Sozialordnung und dem Deutschen Bundesarchiv, Band 1: Grundlagen, Baden-Baden.

Koslowski, P. (Hg., 1999), Das Gemeinwohl zwischen Universalismus und Partikularismus, StuttgartBad Cannstadt.

Leubuscher, C. (1923), Die Krise der Sozialpolitik, in: Soziale Praxis 32, Sp. 339-343; 363-366; 387-390.

Lüscher, K. (1997), Solidarische Beziehungen: das ,neue' Problem der Generationen, in: Modernität und Solidarität. Konsequenzen gesellschaftlicher Modernisierung, hg. v. K. Gabriel u. a., Freiburg i. Br., S. 59-77. 
Luhmann, Niklas $\left(1999^{4}\right)$, Grundrechte als Institution. Ein Beitrag zur politischen Soziologie [1965], Berlin.

Luhmann, N. (1968), Vertrauen - Ein Mechanismus der Reduktion sozialer Komplexität, Stuttgart.

Luhmann, N. (1972), Einfache Sozialsysteme, in: Zeitschrift für Soziologie 1, S. 51-65.

Luhmann, N. (1978), Soziologie der Moral, in: Theorietechnik und Moral, hg. v. N. Luhmann u. S. H. Pfürtner, Frankfurt/M., S. 8-116.

Luhmann, N. (1990), Paradigm lost: Über die ethische Reflexion der Moral.

Luhmann, N. (2000), Die Politik der Gesellschaft, Frankfurt/M.

Marshall T. H. (1992), Social Class and Citizenship [1949], dt. in: Bürgerrechte und soziale Klassen. Zur Soziologie des Wohlfahrtsstaates, hg. v. Elmar Rieger, Frankfurt/M./New York, S. 33-94.

Mauss, M. (1968), Die Gabe: Form und Funktion des Austauschs in archaischen Gesellschaften [1923], Frankfurt/M.

Meyer, A. (1864), Zum Begriffe der Socialpolitik, in: Preußische Jahrbücher 14, S. 315-330.

Münkler, H./Fischer, K. (1999), Gemeinwohl und Gemeinsinn. Thematisierung und Verbrauch soziomoralischer Ressourcen in der modernen Gesellschaft, in: Berlin-Brandenburgische Akademie der Wissenschaften: Berichte und Abhandlungen, Band 7, Berlin, S. 237-265.

Pankoke, E. (1970), Sociale Bewegung - Sociale Frage - Sociale Politik. Grundifragen der deutschen „Socialwissenschaft“ im 19. Jahrhundert, Stuttgart.

Pankoke, E. (Hg., 1991), Gesellschaftslehre, Frankfurt/M.

Parsons, T. (1972), Das System moderner Gesellschaften, München.

Phelps, E. S. (Hg., 1975), Altruism, Morality, and Economic Theory, New York.

Preller, L. (1962), Sozialpolitik - Theoretische Ortung, Tübingen/Zürich.

Putnam, R. D. (1995), Bowling Alone: America's Declining Social Capital, in: Journal of Democracy 6, S. $65-78$.

Schmoller, G. (1864), Die Arbeiterfrage, in: Preußische Jahrbücher, 14, S. 393-424 (I); S. 523-547 (II); 15 (1865), S. 32-63 (III).

Schmoller, G. (1998a), Die sociale Frage und der preußische Staat [1874], in: G. Schmoller, (1998b), Historisch-ethische Nationalökonomie als Kulturwissenschaft. Ausgewählte methodologische Schriften, hg. v. H. H. Nau, Marburg S. 75-96.

Schmoller, G. (1998b), Historisch-ethische Nationalökonomie als Kulturwissenschaft. Ausgewählte methodologische Schriften, hg. v. H. H. Nau, Marburg.

Schulze, H.-J./Künzler, J. (1997), Familie und Modernisierung: Kein Widerspruch, in: Modernität und Solidarität. Konsequenzen gesellschaftlicher Modernisierung, hg. v. K. Gabriel u. a., Freiburg i. Br., S. 91-105.

Schweitzer, R. v. (1997), Geschlechtersolidarität und Modernität in der Familienforschung, in: Modernität und Solidarität. Konsequenzen gesellschaftlicher Modernisierung, hg. v. K. Gabriel u. a., Freiburg i. Br., S. 29-57.

Siep, L. (1992), Verfassung, Grundrechte und soziales Wohl in Hegels Philosophie des Rechts, in: Praktische Philosophie im Deutschen Idealismus, hg. v. L. Siep, Frankfurt/M.

Sinzheimer, H. $\left(1977^{2}\right)$, Ein Arbeitstarifgesetz. Die Idee der sozialen Selbstbestimmung im Recht [1916], München/Leipzig.

Sinzheimer, H. (1976), Arbeitsrecht und Rechtssoziologie. Gesammelte Aufsätze und Reden, hg. v. O. Kahn-Freund u. T. Ramm, 2 Bde, Frankfurt/M./Köln.

Smith, A. (1977), Theorie der ethischen Gefühle, hg. v. Walther Eckstein, Hamburg.

Stein, L. v. (1972), Geschichte der sozialen Bewegung in Frankreich von 1789 bis auf unsere Tage [1850], 3 Bände., Darmstadt.

Thiemeier, T. (1988), Wirtschaftspolitik als Wissenschaft. Gerhard Weissers System der Politik aus normativen Grundentscheidungen, in: Sozialer Fortschritt 37, S.73-78.

Tönnies, F. (1970), Gemeinschaft und Gesellschaft [1887], Darmstadt. 
Treitschke, H. v. (1859), Die Gesellschaftswissenschaft. Ein kritischer Versuch, Leipzig.

Ulrich, G. (1994), Politische Steuerung. Staatliche Intervention aus systemtheoretischer Sicht, Opladen.

Vobruba, G. (1994), Gemeinschaft ohne Moral. Theorie und Empirie moralfreier Gemeinschaftskonstruktionen, Wien.

Weber, M. (1964), Wirtschaft und Gesellschaft. Grundriss der verstehenden Soziologie. Studienausgabe, hg. v. J. Winkelmann, Köln/Berlin.

Weisser, G. (1959), Distribution (II) Politik, in: Handwörterbuch der Sozialwissenschaften, Bd. 2, Stuttgart u. a., S. 635-654.

Wiesenthal, H. (2000), Markt, Organisation und Gemeinschaft als ,zweitbeste ${ }^{6}$ Verfahren sozialer Koordination, in: Gesellschaftliche Komplexität und kollektive Handlungsfähigkeit, hg. v. Raymund Werle u. Uwe Schimank, Frankfurt/M./New York, S. 44-73.

Willke, H. (1995), Systemtheorie III: Steuerungstheorie, Stuttgart/Jena.

Zahlmann, C. (Hg., 1992), Kommunitarismus in der Diskussion: eine streitbare Einführung, Berlin.

Zoll, R. (2000), Was ist Solidarität heute?, Frankfurt/M. 